Algebraic $8 \mathcal{G}$ Geometric $\mathcal{T}_{\text {opology }}$

Volume 5 (2005) 71-106

Published: 3 February 2005

ATG

\title{
Clover calculus for homology 3-spheres via basic algebraic topology
}

\author{
Emmanuel Auclair \\ Christine Lescop
}

\begin{abstract}
We present an alternative definition for the Goussarov-Habiro filtration of the $\mathbb{Z}$-module freely generated by oriented integral homology 3 -spheres, by means of Lagrangian-preserving homology handlebody replacements (LP-surgeries). Garoufalidis, Goussarov and Polyak proved that the graded space $\left(\mathcal{G}_{n}\right)_{n}$ associated to this filtration is generated by Jacobi diagrams. Here, we express elements associated to LP-surgeries as explicit combinations of these Jacobi diagrams in $\left(\mathcal{G}_{n}\right)_{n}$. The obtained coefficient in front of a Jacobi diagram is computed like its weight system with respect to a Lie algebra equipped with a non-degenerate invariant bilinear form, where cup products in 3-manifolds play the role of the Lie bracket and the linking number replaces the invariant form. In particular, this article provides an algebraic version of the graphical clover calculus developed by Garoufalidis, Goussarov, Habiro and Polyak. This version induces splitting formulae for all finite type invariants of homology 3-spheres.
\end{abstract}

AMS Classification 57M27; 57N10

Keywords 3-manifolds, homology spheres, finite type invariants, Jacobi diagrams, Borromeo surgery, clover calculus, clasper calculus, GoussarovHabiro filtration

\section{Introduction}

In 1995, in Oht, Tomotada Ohtsuki introduced a notion of finite type invariants for homology 3-spheres (that are compact oriented 3-manifolds with the same homology with integral coefficients as the standard 3 -sphere $S^{3}$ ), following the model of the theory of Vassiliev invariants for knots in the ambient space $\mathbb{R}^{3}$. He defined a filtration of the real vector space freely generated by homology 3-spheres and began the study of the associated graded space. In [Le, Thang Le finished identifying this graded space to a space of Jacobi diagrams called $\mathcal{A}_{\mathbb{R}}(\emptyset)$. The Jacobi diagrams, precisely defined in Subsection 2.1 are represented by trivalent finite graphs with additional orientation information. 
Similar filtrations of the $\mathbb{Z}$-module freely generated by homology 3 -spheres and their relationships have been studied by Garoufalidis, Goussarov, Polyak and others. See GGP] and references therein. Over $\mathbb{Z}[1 / 2]$, all of them are equivalent to the Ohtsuki filtration GGP.

Among these filtrations, the most convenient one is the Goussarov-Habiro one where the Matveev Borromeo surgeries Mat (defined in Subsection 2.2) play the role of the crossing changes in the knot case. It allowed Garoufalidis, Goussarov and Polyak to define a set of generators $\Psi_{n}(\Gamma)$ for the degree $n$ part $\mathcal{G}_{n}$ of the associated Goussarov-Habiro graded $\mathbb{Z}$-module, for Jacobi diagrams $\Gamma$ with at most $n$ vertices GGP. See Subsection 2.3. Garoufalidis, Goussarov and Polyak also gave some graphical rules that allow one to reduce an element to a combination of their generators. This set of rules is the so-called clover calculus. Here, these rules are enclosed in two propositions 4.13 and $\mathbf{A . 1}$

Our main theorem 3.5 expresses elements of $\mathcal{G}_{n}$ associated to the $L P$-surgeries defined in Subsection 3.1] as explicit combinations of the $\Psi_{n}(\Gamma)$, in terms of intersection forms (or cup products) and linking numbers. Therefore, this article presents a completely algebraic version of the Garoufalidis-Goussarov-HabiroPolyak clover calculus. Furthermore, it tightens the links between Jacobi diagrams and topology by relating the vertices of the Jacobi diagrams to cup products in 3 -manifolds and the diagram edges with linking numbers.

We also give an alternative definition of the Goussarov-Habiro filtration of the $\mathbb{Z}$-module of integral homology 3 -spheres, by means of LP-surgeries. See Corollary 3.2 .

Let us now give a slightly more specific description of our main theorem 3.5

A homology genus $g$ handlebody is an oriented compact 3-manifold with the same integral homology as the standard genus $g$ handlebody $H_{g}$. The boundary $\partial A$ of such a manifold $A$ is then homeomorphic to the genus $g$ surface $\partial H_{g}$. The Lagrangian $\mathcal{L}_{A}$ of $A$ is the kernel of the map induced by the inclusion from $H_{1}(\partial A ; \mathbb{Z})$ to $H_{1}(A ; \mathbb{Z})$. A Lagrangian-preserving surgery or LP-surgery on a homology sphere $M$ consists in removing the interior of such a homology handlebody $(A \subset M)$ and replacing it by another such $B$ whose boundary $\partial B$ is identified to $\partial A$ so that $\mathcal{L}_{A}=\mathcal{L}_{B}$.

In our definition of the Goussarov-Habiro filtration $\left(\mathcal{F}_{n}\right)_{n \in \mathbb{N}}$ of the $\mathbb{Z}$-module $\mathcal{F}=\mathcal{F}_{0}$ freely generated by the oriented homology spheres up to orientationpreserving homeomorphisms, the $n^{\text {th }}$ module $\mathcal{F}_{n}$ is generated by brackets $[D]$ of so-called $n$-component LP-surgeries $D$ that are made of $n$ disjoint LP- 
surgeries $\left(A_{i}, B_{i}\right)$ in $M$. (The $A_{i}$ are disjoint in $M$.)

$$
[D]=\sum_{J \subset\{1, \ldots, n\}}(-1)^{\sharp J}\left(\left(M \backslash \sqcup_{j \in J} \operatorname{Int}\left(A_{j}\right)\right) \cup_{\partial}\left(\sqcup_{j \in J} B_{j}\right)\right) \text {. }
$$

Our main result expresses the bracket $[D]$ of an $n$-component LP-surgery $D$ as

$$
[D]=\sum_{\Gamma} \ell(D ; \Gamma) \Psi_{n}(\Gamma)
$$

in $\mathcal{G}_{n}=\mathcal{F}_{n} / \mathcal{F}_{n+1}$, where the coefficient $\ell(D ; \Gamma)$ of $\Psi_{n}(\Gamma)$ is an explicit function of the cup products in the manifolds $\left(A_{i} \cup-B_{i}\right)$, of the linking pairings on $H_{1}\left(A_{i}\right) \otimes H_{1}\left(A_{j}\right), i \neq j$, and of variations of the Rohlin invariant when replacing $A_{i}$ by $B_{i}$.

Let us roughly define $\ell(D ; \Gamma)$ when $n$ is the number of vertices of $\Gamma$ and when $\Gamma$ admits no non-trivial automorphism. The general definition of $\ell(D ; \Gamma)$ is given in Subsection 3.2. When a bijection $\sigma$ from the set of vertices of $\Gamma$ to $\{1, \ldots, n\}$ is given, the algebraic intersection of surfaces (or the cup product) of each $\left(A_{i} \cup-B_{i}\right)$ is placed at the vertex $\sigma^{-1}(i)$. The cup products are next contracted along the edges with respect to the linking pairing to produce a number $\ell(D ; \Gamma ; \sigma)$, and $\ell(D ; \Gamma)=\sum_{\sigma} \ell(D ; \Gamma ; \sigma)$. This construction is similar to the construction of weight systems associated to Lie algebras.

The proof of Theorem 3.5 goes as follows. We first prove that the standard Goussarov, Garoufalidis and Polyak generators have appropriate coefficients in Subsection 4.1. Then we use the similarities between the behaviour of the bracket in $\mathcal{G}_{n}$ and the behaviour of our coefficients to reduce the proof to this former case.

Though this article is largely inspired by GGP], it is written in a self-contained way in an attempt to replace all the graphical arguments in GGP by more intrinsic arguments of geometric or algebraic topology.

Theorem 3.5 can be used to derive formulae on the behaviour under LPsurgeries of all finite-type invariants of homology spheres in the GoussarovHabiro sense. For example, it immediately leads to splitting formulae for the restriction to homology spheres of the Kontsevich-Kuperberg-Thurston universal finite-type invariant $Z_{K K T}$ of rational homology spheres. In [L2], the second author proved that these formulae generalise to rational homology spheres and to rational homology handlebody replacements that preserve the rational Lagrangians. These generalized splitting formulae are fairly easy to guess from the Kontsevich-Kuperberg-Thurston construction (but much harder to prove in general), they actually led the second author to the formulae of Theorem 3.5 . 
These formulae had been previously noticed by G. Kuperberg and D. Thurston in the special case where rational homology handlebodies are reglued by a homeomorphism that induces the identity in homology [KT]. This special case is sufficient to prove that $Z_{K K T}$ is universal among finite-type invariants of homology spheres.

The second author thanks Thang Le, Gregor Masbaum and Dylan Thurston for useful and pleasant conversations.

\section{Background}

\subsection{Jacobi diagrams}

In what follows, a Jacobi diagram $\Gamma$ is a trivalent graph without simple loop like $-\infty$. Let $V(\Gamma)$ and $E(\Gamma)$ denote the set of vertices of $\Gamma$ and the set of edges of $\Gamma$, respectively. A half-edge $c$ of $\Gamma$ is a pair $c=(v(c) ; e(c))$ where $v(c) \in V(\Gamma), e(c) \in E(\Gamma)$ and $v(c)$ belongs to $e(c)$. The set of half-edges of $\Gamma$ will be denoted by $H(\Gamma)$ and its two natural projections above onto $V(\Gamma)$ and $E(\Gamma)$ will be denoted by $v$ and $e$, respectively. An automorphism of a Jacobi diagram $\Gamma$ is a permutation $\phi$ of $H(\Gamma)$ verifying the two following conditions

$$
\begin{aligned}
& \left(e(c)=e\left(c^{\prime}\right)\right) \Rightarrow\left(e(\phi(c))=e\left(\phi\left(c^{\prime}\right)\right)\right) \\
& \left(v(c)=v\left(c^{\prime}\right)\right) \Rightarrow\left(v(\phi(c))=v\left(\phi\left(c^{\prime}\right)\right)\right)
\end{aligned}
$$

for any $c, c^{\prime} \in H(\Gamma)$. An automorphism $\phi$ of a Jacobi diagram $\Gamma$ preserves the vertices of $\Gamma$ if

$$
\forall c \in H(\Gamma), v(\phi(c))=v(c) .
$$

Let $\operatorname{Aut}(\Gamma)$ be the set of automorphisms of $\Gamma$. Let $\operatorname{Aut}_{V}(\Gamma)$ denote the set of automorphims of $\Gamma$ that preserve the vertices of $\Gamma$. Let $\sharp \operatorname{Aut}_{V}(\Gamma)$ denote the number of automorphisms of $\Gamma$ that preserve the vertices. A vertex-orientation of a Jacobi diagram $\Gamma$ is an orientation of each vertex of $\Gamma$, that is a cyclic order of the three half-edges that meet at that vertex. Two vertex-orientations of $\Gamma$ are equivalent if and only if the cardinality of the set of vertices where they differ is even. An orientation of $\Gamma$ is an equivalence class of vertex-orientations. An oriented Jacobi diagram is a Jacobi diagram carrying an orientation. A Jacobi diagram $\Gamma$ is reversible if there exists an automorphism $\phi$ of $\Gamma$ that reverses an orientation of $\Gamma$. For any automorphism $\phi$ of $\Gamma$, set

$$
\operatorname{sign}(\phi)=\left\{\begin{array}{cl}
1 & \text { if } \phi \text { preserves the orientation } \\
-1 & \text { if } \phi \text { reverses the orientation. }
\end{array}\right.
$$


Note that, for all $\phi \in \operatorname{Aut}_{V}(\Gamma), \operatorname{sign}(\phi)=1$. The degree of a Jacobi diagram is half the number of all its vertices. Let $\mathcal{A}_{k}$ denote the free abelian group generated by the degree $k$ oriented Jacobi diagrams, quotiented out by the following relations AS and IHX.

$$
\begin{aligned}
& \dot{\phi}+\boldsymbol{\varphi}^{-}=0 \quad \boldsymbol{h}+\boldsymbol{\phi}+\boldsymbol{\phi}=0 \\
& \text { AS-relation IHX-relation }
\end{aligned}
$$

Each of these relations relate diagrams which can be represented by immersions that are identical outside the part of them represented in the pictures. In the pictures, the cyclic order of the half-edges is represented by the counterclockwise order. For example, AS identifies the sum of two diagrams which only differ by the orientation at one vertex to zero. The space $\mathcal{A}_{0}$ is equal to $\mathbb{Z}$ generated by the empty diagram. In what follows, if $\Gamma$ is an oriented Jacobi diagram, then $-\Gamma$ denotes the same Jacobi diagram with the opposite orientation. If $\Gamma$ is reversible, then $\Gamma=-\Gamma$.

\section{$2.2 \quad Y$-graphs and the Goussarov-Habiro filtration}

Here, we briefly review the $Y$-surgery, or the surgery along $Y$-links, which is presented in GGP. The $Y$-surgery is equivalent to the Borromeo transformation in Matveev's work Mat.

Let $\Lambda$ be the graph embedded in the surface $\Sigma(\Lambda)$ shown in Figure 1(a). In the 3 -handlebody $(N=\Sigma(\Lambda) \times[-1,1])$, the edges of $\Lambda$ are framed by a vector field normal to $\Sigma(\Lambda)=\Sigma(\Lambda) \times\{0\} . \Sigma(\Lambda)$ is called a framing surface for $\Lambda$. Let $L(\Lambda) \subset N$ be the link presented in Figure 1(b) with six framed components that inherit their framings from $\Sigma(\Lambda)$.

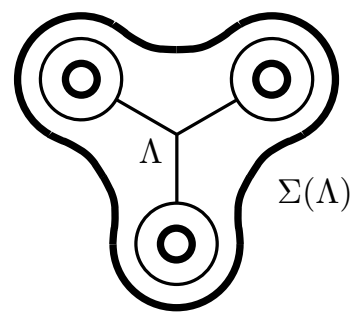

(a)

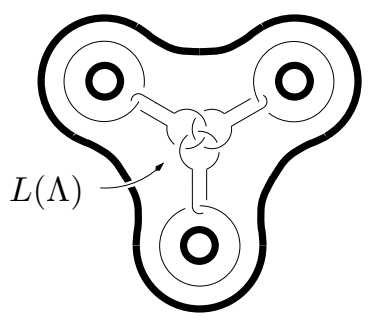

(b)

Figure 1: $Y$-graph and associated link

Let $M$ be a 3-manifold. A $Y$-graph in $M$ is an embedding $\phi$ of $N($ or $\Sigma(\Lambda))$ into $M$ up to isotopy. Such an isotopy class is determined by the framed image 
of the framed unoriented graph $\Lambda$ under $\phi$. A leaf of a $Y$-graph $\phi$ is the image under $\phi$ of a simple loop of our graph $\Lambda$. An edge of $\phi$ is an edge of $\phi(\Lambda)$ that is not a leaf. The vertex of $\phi$ is the unique vertex of $\phi(\Lambda)$ adjacent to the three edges. With this terminology, a $Y$-graph has one vertex, three edges and three leaves:

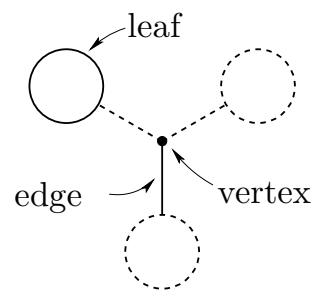

Let $G \subset M$ be a $Y$-graph. A leaf $l$ of a $Y$-component of $G$ is trivial if $l$ bounds an embedded disc that induces the framing of $l$, in $M \backslash G$.

The $Y$-surgery along the $Y$-graph $\phi(\Lambda)$ is the surgery along the framed link $\phi(L(\Lambda))$ (see [Rol, Chapter 9], or [Lic, Chapter 11] for details about surgery on framed knots). The resulting manifold is denoted by $M_{\phi(\Lambda)}$. An $n$-component $Y$-link $G \subset M$ is an embedding of the disjoint union of $n$ copies of $N$ into $M$ up to isotopy. The $Y$-surgery along a $Y$-link $G$ is defined as the surgery along each $Y$-component of $G$. The resulting manifold is denoted by $M_{G}$.

In this article, the homology coefficients will always be integers. A $\mathbb{Z}$-sphere is a compact oriented 3-manifold $M$ such that $H_{*}(M)=H_{*}\left(S^{3}\right)$. It is also called a homology sphere. A homology handlebody or $\mathbb{Z}$-handlebody is an oriented, compact 3-manifold $A$ with the same homology (with integral coefficients) as the standard (solid) handlebody $H_{g}$ below.

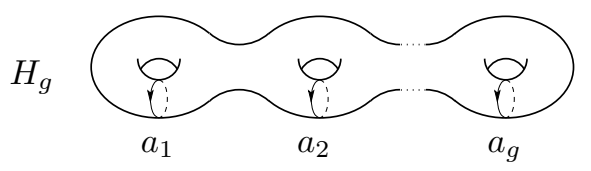

Note that the boundary $\partial A$ of such a $\mathbb{Z}$-handlebody $A$ is homeomorphic to the boundary $\Sigma_{g}$ of $H_{g}$. For any surface $\Sigma$, let $\langle,\rangle_{\Sigma}$ be the intersection form on $H_{1}(\Sigma)$. For a $\mathbb{Z}$-handlebody $A, \mathcal{L}_{A}$ denotes the kernel of the map from $H_{1}(\partial A)$ to $H_{1}(A)$ induced by the inclusion. It is a Lagrangian of $\left(H_{1}(\partial A) ;\langle,\rangle_{\partial A}\right)$. It is called the Lagrangian of $A$.

If $A$ is a $\mathbb{Z}$-handlebody and if $G$ is a $Y$-link in the interior $\operatorname{Int}(A)$ of $A$, then $A_{G}$ is still a $\mathbb{Z}$-handlebody whose boundary $\partial A$ is canonically identified with 
$\partial A_{G}$, so that $\mathcal{L}_{A}=\mathcal{L}_{A_{G}}$. Similarly, if $G$ is a $Y$-link in a homology sphere $M$, then $M_{G}$ is still a homology sphere.

Let $\mathcal{F}$ be the abelian group freely generated by the oriented $\mathbb{Z}$-spheres up to orientation-preserving diffeomorphisms. Let $M$ be a $\mathbb{Z}$-sphere and let $G \subset M$ be a $Y$-link with $n$ components indexed by $\{1, \ldots, n\}$. For any subset $J \subset$ $\{1, \ldots, n\}$, let $G(J)$ be the $Y$-sublink of $G$ made of the components of $G$ whose indices are in $J$. Set

$$
[M, G]=\sum_{J \subset\{1, \ldots, n\}}(-1)^{\sharp J} M_{G(J)} \in \mathcal{F} .
$$

Let $\mathcal{F}_{n}$ denote the subgroup of $\mathcal{F}$ generated by all the elements $[M, G]$, where $G$ is an $n$-component $Y$-link in a $\mathbb{Z}$-sphere $M$. This defines a filtration

$$
\mathcal{F}_{0}=\mathcal{F} \supset \mathcal{F}_{1} \supset \ldots \mathcal{F}_{n} \supset \ldots
$$

of $\mathcal{F}$. It is called the Goussarov-Habiro filtration (see [GGP] and [Hbo]). Set

$$
\mathcal{G}_{n}=\mathcal{F}_{n} / \mathcal{F}_{n+1}
$$

\subsection{Linking Jacobi diagrams to the Goussavov-Habiro filtra- tion}

Below, following GGP, we describe a surjective map from $\oplus_{2 k \leq n} \mathcal{A}_{k}$ to $\mathcal{G}_{n}$, whose tensor product by $\mathbb{Z}[1 / 2]$ is an isomorphism. Let $k$ and $n$ be integers such that $2 k \leq n$. Let $\Gamma$ be a degree $k$ oriented Jacobi diagram. Let $\tilde{\Gamma}$ be an arbitrary framed embedding of $\Gamma$ in $S^{3}$, where the framing is induced by a regular projection of $\tilde{\Gamma}$ in $\mathbb{R}^{2}$ that induces the counterclockwise orientation of the trivalent vertices of $\Gamma$. Insert a Hopk link on each edge of $\tilde{\Gamma}$ as illustrated in Figure 2(a), Let $G(\tilde{\Gamma})$ denote the resulting $Y$-link in $S^{3}$.

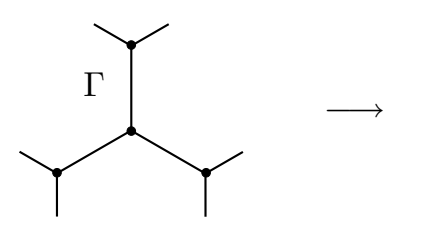

(a)

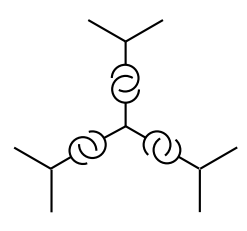

Figure 2: Turning a Jacobi diagram into a $Y$-link

Let $Y_{I I I}$ be the framed $Y$-graph embedded in $S^{3}$ shown in Figure 2(b), Let $\phi_{n}(\Gamma)$ be the disjoint union of $G(\tilde{\Gamma})$ and of $n-2 k$ copies of $Y_{I I I}$. 
Theorem 2.1 GGP Theorem 4.13] The linear map

$$
\begin{aligned}
\Psi_{n}: \bigoplus_{2 k \leq n} \mathcal{A}_{k} & \longrightarrow \frac{\mathcal{G}_{n}}{\Gamma}
\end{aligned}
$$

does not depend on the choice of $\phi_{n}$, is well-defined and is surjective. Moreover, in $\mathcal{G}_{n}$,

$$
2 \Psi_{n}\left(\bigoplus_{2 k<n} \mathcal{A}_{k}\right)=0
$$

That $\Psi_{n}$ is independent of the choice of $\phi_{n}$, factors through $A S$ and satisfies $2 \Psi_{n}\left(\oplus_{2 k<n} \mathcal{A}_{k}\right)=0$ is a consequence of Proposition 4.13 proved below. In this article, the class $\overline{[M, G]} \in \mathcal{G}_{n}$ of the bracket $[M, G]$ of any $n$-component $Y$-link, will be expressed as an explicit combination of the $\Psi_{n}(\Gamma)$ for oriented Jacobi diagrams $\Gamma$ with at most $n$ vertices. Therefore, the surjectivity of $\Psi_{n}$ will be reproved. For the sake of completeness, a proof that $\Psi_{n}$ factors through IHX is given in the appendix.

\section{Statement of the main result}

\section{$3.1 \quad$ LP-surgeries}

An $n$-component LP-surgery is a 3 -tuple

$$
D=\left(M ; n ;\left(A_{i}, B_{i}\right)_{i=1, \ldots, n}\right)
$$

where

- $M$ is a homology sphere, $n \in \mathbb{N}$,

- for any $i=1,2, \ldots n, A_{i}$ and $B_{i}$ are $\mathbb{Z}$-handlebodies whose boundaries are identified by implicit diffeomorphisms (we shall write $\partial B_{i}=\partial A_{i}$ ), so that $\mathcal{L}_{B_{i}}=\mathcal{L}_{A_{i}}$

- the disjoint union of the $A_{i}$ is embedded in $M$. We shall write

$$
\sqcup_{i=1}^{n} A_{i} \subset M .
$$

For such an LP-surgery $D$, and for any subset $J \subset\{1, \ldots, n\}$, set

$$
D(J)=\left(M ; \sharp J ;\left(A_{i}, B_{i}\right)_{i \in J}\right) .
$$

Let $M_{D(J)}$ denote the homology sphere obtained by replacing $A_{i}$ by $B_{i}$ for any element $i$ of $J$.

$$
M_{D(J)}=\left(M \backslash \sqcup_{i \in J} \operatorname{Int}\left(A_{i}\right)\right) \bigcup_{\sqcup_{i \in J} \partial A_{i}}\left(\sqcup_{i \in J} B_{i}\right) .
$$


Define

$$
[D]=\sum_{J \subset\{1, \ldots, n\}}(-1)^{\sharp J} M_{D(J)} \in \mathcal{F} .
$$

The following proposition will be proved in Subsection 4.2

Proposition 3.1 For any $n$-component LP-surgery $D$,

$$
[D] \in \mathcal{F}_{n} .
$$

Conversely, any $n$-component $Y$-link $G=\left(G_{i}\right)_{i \in\{1, \ldots, n\}}$ in a homology sphere $M$, induces the $n$-component LP-surgery

$$
(M ; G)=\left(M ; n ;\left(A_{i}, B_{i}\right)_{i=1, \ldots, n}\right)
$$

such that, for any $i$ in $\{1, \ldots, n\}, A_{i}$ is a regular neighbourhood of the $Y-$ component $G_{i}$ of $G$, and $B_{i}=\left(A_{i}\right)_{G_{i}}$. Then $[(M ; G)]=[M, G]$ and $(M ; G)$ is called the LP-surgery induced by $G$.

This allows us to give the following alternative definition for the GoussarovHabiro filtration.

Corollary 3.2 $\mathcal{F}_{n}$ is the subspace of $\mathcal{F}$ generated by the elements $[D]$, where $D$ runs among the $n$-component $L P$-surgeries.

In what follows, for any $n$-component LP-surgery $D, \overline{[D]}$ denotes the class of $[D]$ in $\mathcal{G}_{n}$. It is called the bracket of $D$.

\subsection{The linking number of an LP-surgery with respect to a Jacobi diagram}

This subsection is devoted to the definition of the linking number $\ell(D ; \Gamma)$ of an $n$-component LP-surgery $D$ with respect to a degree $k$ Jacobi diagram $\Gamma$, with $2 k \leq n$.

Let $\Gamma$ be an oriented degree $k$ Jacobi diagram. Define a map

$$
h: H(\Gamma) \longrightarrow\{1,2,3\}
$$

such that, for any vertex $w$ of $\Gamma$, the map

$$
h_{w}: v^{-1}(w) \longrightarrow\{1,2,3\}
$$


is a bijection. Set

$$
\operatorname{sign}(h)=\prod_{w \in V(\Gamma)} \operatorname{sgn}\left(h_{w}\right)
$$

where, for any vertex $w$ of $\Gamma, \operatorname{sgn}\left(h_{w}\right)=1$ if the orientation of $w$ is induced by the order of the half-edges given by $h_{w}$, and $\operatorname{sgn}\left(h_{w}\right)=-1$ otherwise. A coloration of $\Gamma$ is a bijection $\sigma: V(\Gamma) \longrightarrow\{1, \ldots, 2 k\}$. Below, $\sigma$ also denotes the induced map $\sigma \circ v: H(\Gamma) \longrightarrow\{1, \ldots, 2 k\}$. Let $D=\left(M ; 2 k ;\left(A_{i}, B_{i}\right)\right)$ be a $2 k$-component LP-surgery. Let us define the linking number $\ell(D ; \Gamma ; \sigma)$ of $D$ with respect to $\Gamma$ and $\sigma$.

The boundary of an oriented manifold is always oriented with the outward normal first convention. The Mayer-Vietoris boundary map

$$
\partial_{i, M V}: H_{2}\left(A_{i} \cup_{\partial A_{i}}-B_{i}\right) \longrightarrow \mathcal{L}_{A_{i}},
$$

that maps the homology class of an oriented surface to the oriented boundary of its intersection with $A_{i}$, is an isomorphism. This isomorphism carries the triple intersection of surfaces in the closed 3 -manifold $\left(A_{i} \cup_{\partial A_{i}}-B_{i}\right)$ on $\bigotimes^{3} H_{2}\left(A_{i} \cup_{\partial A_{i}}-B_{i}\right)$ to a linear form $\mathcal{I}\left(A_{i}, B_{i}\right)$ on $\bigotimes_{j=1}^{3} \mathcal{L}_{A_{i}}^{(j)}$ which is antisymmetric with respect to the permutation of two factors, where $\mathcal{L}_{A_{i}}^{(j)}$ denotes the $j^{\text {th }}$ copy of $\mathcal{L}_{A_{i}}$. Then the linear form $\mathcal{I}\left(A_{i}, B_{i}\right)$ is an element of $\bigotimes_{j=1}^{3}\left(\mathcal{L}_{A_{i}}^{(j)}\right)^{*}$ where $\left(\mathcal{L}_{A_{i}}^{(j)}\right)^{*}$ denotes the dual $\operatorname{Hom}\left(\mathcal{L}_{A_{i}}^{(j)} ; \mathbb{Z}\right)$ of $\mathcal{L}_{A_{i}}^{(j)}$. Let $c \in H(\Gamma)$. Define

$$
X(c)=\left(\mathcal{L}_{A_{\sigma(c)}}^{(h(c))}\right)^{*} .
$$

The linear form $\mathcal{I}\left(A_{i}, B_{i}\right)$ belongs to

$$
\bigotimes_{(\Gamma) ; \sigma(c)=i\}} X(c)
$$

Then define

$$
T(D ; \Gamma ; \sigma)=\operatorname{sign}(h) \bigotimes_{w \in V(\Gamma)} \mathcal{I}\left(A_{\sigma(w)}, B_{\sigma(w)}\right) \in \bigotimes_{c \in H(\Gamma)} X(c) .
$$

Note that $T(D ; \Gamma ; \sigma)$ is independent of $h$.

Notation 3.3 Let $A$ be a $\mathbb{Z}$-handlebody. Then $H_{1}(A)$ is canonically isomorphic to $\frac{H_{1}(\partial A)}{\mathcal{L}_{A}}$. Furthermore, the intersection form $\langle,\rangle_{\partial A}$ induces the map

$$
\begin{aligned}
& \langle, .\rangle: \quad H_{1}(\partial A) \longrightarrow \mathcal{L}_{A}^{*} \\
& x \quad \longmapsto\langle., x\rangle
\end{aligned}
$$


that in turn induces an isomorphism from $\frac{H_{1}(\partial A)}{\mathcal{L}_{A}}$ to $\mathcal{L}_{A}^{*}$. Then

$$
\varphi_{A}: H_{1}(A) \longrightarrow \mathcal{L}_{A}^{*}
$$

will denote the composition of these two isomorphisms.

For $\{i, j\} \subset\{1,2, \ldots, 2 k\}$, the linking number in $M$ induces a bilinear form on $H_{1}\left(A_{i}\right) \times H_{1}\left(A_{j}\right)$ that is viewed as a linear form on $\mathcal{L}_{A_{i}}^{*} \otimes \mathcal{L}_{A_{j}}^{*}$ via $\varphi_{A_{i}}^{-1} \otimes \varphi_{A_{j}}^{-1}$. Therefore, for each edge $f \in E(\Gamma)$ made of two half-edges $c$ and $d$ (such that $\left.e^{-1}(f)=\{c, d\}\right)$, the linking number yields a contraction

$$
\ell_{f}: X(c) \otimes X(d) \longrightarrow \mathbb{Z}
$$

Applying all these contractions to the tensor $T(D ; \Gamma ; \sigma)$ maps $T(D ; \Gamma ; \sigma)$ to the integral linking number $\ell(D ; \Gamma ; \sigma)$ of $D$ with respect to $\Gamma$ and $\sigma$.

For any automorphism $\phi$ in $\operatorname{Aut}(\Gamma)$, let

$$
\phi_{v}: V(\Gamma) \longrightarrow V(\Gamma)
$$

denote the bijection such that $v \circ \phi=\phi_{v} \circ v$. Let $\operatorname{Bij}(\Gamma)$ denote the set of colorations of $\Gamma$. Then $\operatorname{Aut}(\Gamma)$ acts on $\operatorname{Bij}(\Gamma)$ by the action

$$
\phi \cdot \sigma=\sigma \circ\left(\phi_{v}\right)^{-1} \text {. }
$$

Let $\operatorname{Bij}(\Gamma) / \operatorname{Aut}(\Gamma)$ denote the quotient of $\operatorname{Bij}(\Gamma)$ under this action. Note that, for any automorphism $\phi$ of $\Gamma$,

$$
\ell(D ; \Gamma ; \sigma)=\operatorname{sign}(\phi) . \ell(D ; \Gamma ; \phi . \sigma) .
$$

The following lemma is proved at the end of the next subsection.

Lemma 3.4 There exists an integer $\ell_{0}(D ; \Gamma ; \sigma)$ such that

$$
\ell(D ; \Gamma ; \sigma)=\sharp \operatorname{Aut}_{V}(\Gamma) . \ell_{0}(D ; \Gamma ; \sigma) .
$$

In what follows, for any $\mathbb{Z}$-sphere $M, \mu(M) \in \mathbb{Z} / 2 \mathbb{Z}$ will denote the Rohlin invariant of $M$ that is the reduction mod 2 of the Casson invariant (see GM, Proposition 1.3, Definition 1.6]).

For any $n$-component LP-surgery $D=\left(M ; n ;\left(A_{i}, B_{i}\right)\right)$ and for any subset $J \subset\{1, \ldots, n\}$, set

$$
\mathcal{L}(D(\bar{J}))=\prod_{i \in(\{1, \ldots, n\} \backslash J)}\left(\mu\left(\left(M \backslash \operatorname{Int}\left(A_{i}\right)\right) \cup B_{i}\right)-\mu(M)\right) .
$$

Let $\Gamma$ be an oriented degree $k$ Jacobi diagram. Let $D=\left(M ; n ;\left(A_{i}, B_{i}\right)\right)$ be an $n$-component LP-surgery with $2 k \leq n$. Here, we define the linking number $\ell(D ; \Gamma)$ of $D$ with respect to $\Gamma$. 
- If $2 k=n$ and if $\Gamma$ is not reversible, then set

$$
\ell(D ; \Gamma)=\sum_{\sigma \in \operatorname{Bij}(\Gamma)} \frac{\ell(D ; \Gamma ; \sigma)}{\sharp \operatorname{Aut}(\Gamma)} \in \mathbb{Z} .
$$

Note that

$$
\ell(D ; \Gamma)=\sum_{\bar{\sigma} \in \operatorname{Bij}(\Gamma) / \operatorname{Aut}(\Gamma)} \ell_{0}(D ; \Gamma ; \sigma) .
$$

- If $2 k=n$ and if $\Gamma$ is reversible, then set

$$
\ell(D ; \Gamma)=\sum_{\bar{\sigma} \in \operatorname{Bij}(\Gamma) / \operatorname{Aut}(\Gamma)} \overline{\ell_{0}(D ; \Gamma ; \sigma)} \in \mathbb{Z} / 2 \mathbb{Z}
$$

where $\overline{\ell_{0}(D ; \Gamma ; \sigma)} \in \mathbb{Z} / 2 \mathbb{Z}$ denotes the mod 2 reduction of $\ell_{0}(D ; \Gamma ; \sigma)$.

- If $2 k<n$, then set

$$
\ell(D ; \Gamma)=\sum_{\{J \subset\{1, \ldots, n\} ; \sharp J=2 k\}} \ell(D(J) ; \Gamma) \cdot \mathcal{L}(D(\bar{J})) \in \mathbb{Z} / 2 \mathbb{Z} .
$$

\subsection{Expression of brackets of LP-surgeries in terms of Jacobi diagrams}

Let $n \in \mathbb{N}$. Let $\mathcal{J}_{n}$ be a set of oriented Jacobi diagrams of degree at most $n / 2$ that contains one Jacobi diagram in each isomorphism class of non-oriented Jacobi diagrams of degree at most $n / 2$. The main goal of this paper is to show the following result.

Theorem 3.5 Let $D$ be an $n$-component LP-surgery. Then

$$
\overline{[D]}=\sum_{\Gamma \in \mathcal{J}_{n}} \ell(D ; \Gamma) \cdot \Psi_{n}(\Gamma) \in \mathcal{G}_{n} .
$$

Proof of Lemma 3.4 For any $i \in\{1, \ldots, 2 k\}$, let $\left(a_{j}^{i}\right)_{j \in J_{i}}$ be a basis of $\mathcal{L}_{A_{i}}$, where $J_{i}=\left\{1, \ldots, g_{i}\right\}$ and $g_{i}$ is the genus of $\partial A_{i}$. Let $\left(z_{j}^{i}\right)_{j \in J_{i}}$ be the basis of $H_{1}\left(A_{i}\right)$ such that, for any $k$ and $l$ in $J_{i},\left(\varphi_{A_{i}}\left(z_{k}^{i}\right)\right)\left(a_{l}^{i}\right)=\delta_{k l}$. Let $c_{1}, c_{2}$ and $c_{3}$ be the three half-edges of $\Gamma$ such that $\sigma\left(c_{k}\right)=i$ and $h\left(c_{k}\right)=k$. Then

$$
\mathcal{I}\left(A_{i}, B_{i}\right)=\sum_{\left(j_{1}, j_{2}, j_{3}\right) \in J_{i}^{3}} \mathcal{I}\left(A_{i}, B_{i}\right)\left(a_{j_{1}}^{i}, a_{j_{2}}^{i}, a_{j_{3}}^{i}\right) \varphi_{A_{i}}\left(z_{j_{1}}^{i}\right) \otimes \varphi_{A_{i}}\left(z_{j_{2}}^{i}\right) \otimes \varphi_{A_{i}}\left(z_{j_{3}}^{i}\right)
$$




$$
=\sum_{\substack{\left(j_{1}, j_{2}, j_{3}\right) \in J^{3} \\ j_{1}<j_{2}<j_{3}}}\left(\mathcal{I}\left(A_{i}, B_{i}\right)\left(a_{j_{1}}^{i}, a_{j_{2}}^{i}, a_{j_{3}}^{i}\right) \sum_{\tau \in \mathcal{S}_{3}}\left(\operatorname{sgn}(\tau) \bigotimes_{k=1,2,3} \varphi_{A_{i}}\left(z_{j_{\tau(k)}}^{i}\right)\right)\right)
$$

where $\varphi_{A_{i}}\left(z_{j_{\tau(k)}}^{i}\right) \in X\left(c_{k}\right), \mathcal{S}_{3}$ denotes the set of the permutations of $\{1,2,3\}$ and $\operatorname{sgn}(\tau)$ denotes the signature of the permutation $\tau$.

Let $\mathcal{H}(\Gamma)$ denote the set of maps $h^{\prime}: H(\Gamma) \longrightarrow\{1,2,3\}$ such that $h^{\prime}\left(v^{-1}(w)\right)=$ $\{1,2,3\}$ for any $w \in V(\Gamma)$. Set

$$
J=\left\{\left(j_{1}^{1}, j_{2}^{1}, j_{3}^{1}, \ldots, j_{1}^{2 k}, j_{2}^{2 k}, j_{3}^{2 k}\right) \in \prod_{i=1}^{2 k}\left(J_{i}\right)^{3} ; \forall i \in\{1, \ldots, 2 k\}, j_{1}^{i}<j_{2}^{i}<j_{3}^{i}\right\} .
$$

For any $j \in J$, set $\mathcal{J}(j)=\prod_{i=1}^{2 k} \mathcal{I}\left(A_{i}, B_{i}\right)\left(a_{j_{1}}^{i}, a_{j_{2}}^{i}, a_{j_{3}}^{i}\right)$. Then

$$
T(D ; \Gamma ; \sigma)=\sum_{j \in J} \mathcal{J}(j)\left(\sum_{h^{\prime} \in \mathcal{H}(\Gamma)} \operatorname{sign}\left(h^{\prime}\right) \bigotimes_{c \in H(\Gamma)} \varphi_{A_{\sigma(c)}}\left(\begin{array}{c}
\sigma(c) \\
z_{h_{h^{\prime}(c)}^{\sigma(c)}}^{\sigma(c)}
\end{array}\right)\right) .
$$

Then $\ell(D ; \Gamma ; \sigma)=\sum_{h^{\prime} \in \mathcal{H}(\Gamma)} \ell\left(D ; \Gamma ; \sigma ; h^{\prime}\right)$ where

$$
\ell\left(D ; \Gamma ; \sigma ; h^{\prime}\right)=\operatorname{sign}\left(h^{\prime}\right) \sum_{j \in J} \mathcal{J}(j)\left(\prod_{e=\left(c_{1}, c_{2}\right) \in E(\Gamma)} \ell k\left(z_{j_{h^{\prime}\left(c_{1}\right)}^{\sigma\left(c_{1}\right)}}^{\sigma\left(c_{1}\right)}, z_{\left.j_{h^{\prime}\left(c_{2}\right)}^{\sigma\left(c_{2}\right)}\right)}^{\sigma\left(c_{2}\right)}\right)\right) .
$$

For any automorphism $\zeta \in \operatorname{Aut}_{V}(\Gamma), \ell\left(D ; \Gamma ; \sigma ; h^{\prime} \circ \zeta\right)=\ell\left(D ; \Gamma ; \sigma ; h^{\prime}\right)$. Then $\ell_{0}(D ; \Gamma ; \sigma)$ is the sum of the integers $\ell\left(D ; \Gamma ; \sigma ; h^{\prime}\right)$ running over all classes $\overline{h^{\prime}}$ of $\mathcal{H}(\Gamma) / \operatorname{Aut}_{V}(\Gamma)$.

\section{Proof of the theorem}

\subsection{Proof of Theorem 3.5 for LP-surgeries induced by Jacobi diagrams}

Here we prove Theorem 3.5 when $D=\left(S^{3} ; \phi_{n}\left(\Gamma_{Y}\right)\right)$, where the $Y$-link $\phi_{n}\left(\Gamma_{Y}\right)$ is the image of a Jacobi diagram $\Gamma_{Y}$ under the map $\phi_{n}$ of Subsection 2.3. It is a direct corollary of the proposition below (and of Theorem 2.11).

Proposition 4.1 Let $\Gamma$ be an oriented degree $k$ Jacobi diagram. Let $\Gamma_{Y}$ be an oriented degree $k^{\prime}$ Jacobi diagram. Let $n$ be an integer such that $n \geq$ $\max \left(2 k, 2 k^{\prime}\right)$. Then

$$
\ell\left(\phi_{n}\left(\Gamma_{Y}\right) ; \Gamma\right)= \begin{cases}1 & \text { if } \Gamma_{Y} \cong \Gamma \\ -1 & \text { if } \Gamma_{Y} \cong-\Gamma \\ 0 & \text { if } \Gamma_{Y} \nsucceq \pm \Gamma\end{cases}
$$


where $\Gamma \cong \Gamma^{\prime}$ iff $\Gamma$ and $\Gamma^{\prime}$ are isomorphic as oriented Jacobi diagrams.

Lemma 4.2 Let $G$ be a framed $Y$-graph embedded in the interior of a 3 handlebody $A$ as in Figure 3, Let $B$ be the $\mathbb{Z}$-handlebody obtained by $Y-$ surgery on $A$ along $G$. Let $\left(a_{1}, a_{2}, a_{3}\right) \subset \partial A$ be the oriented curves represented in Figure 3. Then $\left(a_{1}, a_{2}, a_{3}\right)$ is a basis of $\mathcal{L}_{A}=\mathcal{L}_{B}$ and

$$
\left|(\mathcal{I}(A, B))\left(a_{1} \otimes a_{2} \otimes a_{3}\right)\right|=1 .
$$

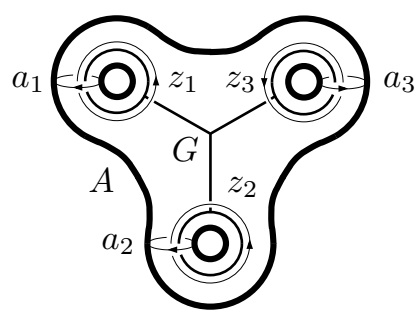

Figure 3

Proof This can be computed directly, or we can use that

$$
A \cup_{\partial A}(-B)=S^{1} \times S^{1} \times S^{1}
$$

$(A \cup(-B)$ is the manifold obtained by surgery on the 0 -framed Borromean link in $S^{3}$ that is $\left(S^{1}\right)^{3}$, see [Thu, 13.1.5]). Let $S_{1}, S_{2}$ and $S_{3}$ be the three following surfaces in $\left(S^{1}\right)^{3}$.

$$
\begin{aligned}
& S_{1}=\{\star\} \times S^{1} \times S^{1} \\
& S_{2}=S^{1} \times\{\star\} \times S^{1} \\
& S_{3}=S^{1} \times S^{1} \times\{\star\} .
\end{aligned}
$$

Let $\mathcal{I} \in\left(\bigotimes^{3} H_{2}\left(\left(S^{1}\right)^{3}\right)\right)^{*}$ be the intersection form of $A \cup_{\partial A}(-B)=\left(S^{1}\right)^{3}$. Since $S_{1} \cap S_{2} \cap S_{3}=\{\star\} \times\{\star\} \times\{\star\}$ is a single transverse intersection point, then

$$
\left|\mathcal{I}\left(S_{1} \otimes S_{2} \otimes S_{3}\right)\right|=1 .
$$

By the isomorphism from $H_{2}\left(\left(S^{1}\right)^{3}\right)$ to $\mathcal{L}_{A}$ induced by the Mayer-Vietoris boundary map, $\left(a_{1}, a_{2}, a_{3}\right)$ can be seen as a basis of $H_{2}\left(\left(S^{1}\right)^{3}\right)$. Therefore, $\mathcal{I}\left(S_{1} \otimes S_{2} \otimes S_{3}\right)$ is a multiple of $(\mathcal{I}(A, B))\left(a_{1} \otimes a_{2} \otimes a_{3}\right)$. Then

$$
\left|(\mathcal{I}(A, B))\left(a_{1} \otimes a_{2} \otimes a_{3}\right)\right|=1 .
$$

Lemma 4.3 $\mu\left(S_{Y_{I I I}}^{3}\right)=1$ 
This lemma is a direct consequence of Corollary 4.21 that is proved in Subssection 4.4. It relies on the results of Subsections 4.2 and 4.3 that are logically independent of the proof below that illustrates our formulae.

\section{Proof of Proposition 4.1}

- First assume that $2 k=2 k^{\prime}=n$.

Let $\sigma$ be a coloration of $\Gamma$. Let $D=\left(S^{3} ; \phi_{n}\left(\Gamma_{Y}\right)\right)=\left(M ; n ;\left(A_{i}, B_{i}\right)\right)$ be the LP-surgery induced by the $Y$-link $\phi_{n}\left(\Gamma_{Y}\right)$. Each pair $\left(A_{i}, B_{i}\right)$ is a copy of the pair $(A, B)$ presented in Lemma 4.2. Let $i \in\{1, \ldots, n\}$. Let $\left(a_{1}^{i}, a_{2}^{i}, a_{3}^{i}\right)$ be the basis of $\mathcal{L}_{A_{i}}$ that corresponds to the curves $\left(a_{1}, a_{2}, a_{3}\right)$ in Figure 3 . Let $\left(z_{1}^{i}, z_{2}^{i}, z_{3}^{i}\right)$ be the basis of $H_{1}\left(A_{i}\right)$ that corresponds to the curves $\left(z_{1}, z_{2}, z_{3}\right)$ in Figure 3. Under the (implicit from now on) isomorphism

$$
\varphi_{A_{i}}: H_{1}\left(A_{i}\right) \longrightarrow \mathcal{L}_{A_{i}}^{*}
$$

presented in Notation 3.3. $\left(z_{1}^{i}, z_{2}^{i}, z_{3}^{i}\right)$ is the dual basis to $\left(a_{1}^{i}, a_{2}^{i}, a_{3}^{i}\right)$, i.e.

$$
\left(\varphi_{A_{i}}\left(z_{k}^{i}\right)\right)\left(a_{l}^{i}\right)=\delta_{k l} \text {. }
$$

Then

$$
\mathcal{I}\left(A_{i}, B_{i}\right)=\sum_{\tau} \operatorname{sgn}(\tau) \mathcal{I}(A, B)\left(a_{1} \otimes a_{2} \otimes a_{3}\right) z_{\tau(1)}^{i} \otimes z_{\tau(2)}^{i} \otimes z_{\tau(3)}^{i} .
$$

Since $\left|(\mathcal{I}(A, B))\left(a_{1} \otimes a_{2} \otimes a_{3}\right)\right|=1$ by Lemma 4.2 ,

$$
T(D ; \Gamma ; \sigma)=\operatorname{sign}(h) \sum_{\left(\tau_{i}\right) \in\left(\mathcal{S}_{3}\right)^{n}}\left(\left(\prod_{i=1}^{n} \operatorname{sgn}\left(\tau_{i}\right)\right) \bigotimes_{c \in H(\Gamma)} z_{\tau_{\sigma(c)}(h(c))}^{\sigma(c)}\right)
$$

where $h$ is as in Subsection 3.2. For any $\tau=\left(\tau_{i}\right)_{i=1, \ldots, n} \in\left(\mathcal{S}_{3}\right)^{n}$, let $\zeta(\sigma ; \tau)$ denote the map

$$
\begin{aligned}
\zeta(\sigma ; \tau): \quad H(\Gamma) & \longrightarrow\{1, \ldots, n\} \times\{1,2,3\} \\
c & \longmapsto\left(\sigma(c), \tau_{\sigma(c)}(h(c))\right) .
\end{aligned}
$$

Let

$$
\begin{aligned}
\xi: H\left(\Gamma_{Y}\right) & \longrightarrow\{1, \ldots, n\} \times\{1,2,3\} \\
c & \longmapsto\left(\xi_{1}(c), \xi_{2}(c)\right)
\end{aligned}
$$

be the bijection such that, for any half-edge $c$ of $\Gamma_{Y}, z_{\xi_{2}(c)}^{\xi_{1}(c)}$ is the core of the leaf corresponding to $c$. Set $\phi(\sigma ; \tau)=\xi^{-1} \circ \zeta(\sigma ; \tau)$. Then $\phi(\sigma ; \tau)$ is a bijection from $H(\Gamma)$ to $H\left(\Gamma_{Y}\right)$ such that

- for any $c, c^{\prime}$ in $H(\Gamma), v(\phi(\sigma ; \tau)(c))=v\left(\phi(\sigma ; \tau)\left(c^{\prime}\right)\right)$ if and only if $v(c)=$ $v\left(c^{\prime}\right)$ 
- for any edge $e=\left(c, c^{\prime}\right)$ of $H(\Gamma)$,

$$
\ell k\left(z_{\tau_{\sigma(c)}^{\sigma(c)}(h(c))}^{\sigma\left(c^{\prime}\right)}, z_{\left.\tau_{\sigma\left(c^{\prime}\right)}^{\sigma\left(h\left(c^{\prime}\right)\right)}\right)}^{\left(c^{\prime}\right)}= \begin{cases}1 & \text { if } \phi(\sigma ; \tau)(c) \text { and } \phi(\sigma ; \tau)\left(c^{\prime}\right) \\ & \text { belong to the same edge of } \Gamma_{Y} \\ 0 & \text { otherwise }\end{cases}\right.
$$

Therefore

$$
\ell(D ; \Gamma ; \sigma)=\sum_{\left\{\tau \in\left(\mathcal{S}_{3}\right)^{n} ; \phi(\sigma ; \tau) \text { is an isomorphism }\right\}} \operatorname{sign}(\phi(\sigma ; \tau))
$$

where

$$
\operatorname{sign}(\phi(\sigma ; \tau))=\operatorname{sign}(h)\left(\prod_{i=1}^{n} \operatorname{sgn}\left(\tau_{i}\right)\right) .
$$

Hence, if $\Gamma \nsubseteq \pm \Gamma_{Y}$, then for any coloration $\sigma$ of $\Gamma, \ell(D ; \Gamma ; \sigma)=0$, and $\ell(D ; \Gamma)=0$.

Otherwise, there exists a coloration $\sigma$ of $\Gamma$ and a map $\tau \in\left(\mathcal{S}^{3}\right)^{n}$ such that $\phi(\sigma ; \tau)$ is an orientation-preserving isomorphism from $\Gamma$ to $\operatorname{sign}(\phi(\sigma ; \tau)) \Gamma_{Y}$. For any map $\tau^{\prime} \in\left(\mathcal{S}^{3}\right)^{n}$ such that $\phi\left(\sigma ; \tau^{\prime}\right)$ is an isomorphism, $\left(\phi\left(\sigma ; \tau^{\prime}\right)\right)^{-1}$ 。 $\phi(\sigma ; \tau)$ is an automorphism of $\Gamma$ that preserves the vertices.

Then $\operatorname{sign}(\phi(\sigma ; \tau))=\operatorname{sign}\left(\phi\left(\sigma ; \tau^{\prime}\right)\right)$.

Conversely, any automorphism of $\operatorname{Aut}_{V}(\Gamma)$ provides such a map $\tau^{\prime}$. Then

$$
\ell(D ; \Gamma ; \sigma)=\operatorname{sign}(\phi(\sigma ; \tau)) \sharp \operatorname{Aut}_{V}(\Gamma) .
$$

For any other pair $\left(\sigma^{\prime} ; \tau^{\prime}\right)$ such that $\phi\left(\sigma^{\prime} ; \tau^{\prime}\right)$ is an isomorphism from $\Gamma$ to $\Gamma_{Y}, \sigma^{\prime}$ is obtained from $\sigma$ by composition by an automorphism of $\Gamma$. Then $\ell(D ; \Gamma)=\ell_{0}(D ; \Gamma ; \sigma)=\operatorname{sign}(\phi(\sigma ; \tau))$ and Proposition 4.1 is proved in this case.

- If $2 k^{\prime}<2 k=n$, then $\ell\left(\phi_{2 k}\left(\Gamma_{Y}\right) ; \Gamma\right)=0$ because when $A$ is the regular neighbourhood of $Y_{I I I}$, the elements of $H_{1}(A)$ do not link any element of the other $H_{1}\left(A_{i}\right)^{\prime}$ 's.

- When $2 k<n$, let $J \subset\{1, \ldots, n\}$ and let $\bar{J}=\{1, \ldots, n\} \backslash J$. Let $J_{Y}$ be the set of indices of the $2 k^{\prime}$-component $Y$-link $G\left(\tilde{\Gamma}_{Y}\right)$. See Subsection 2.3 , $\sharp J_{Y}=2 k^{\prime}$. Set $\overline{J_{Y}}=\{1, \ldots, n\} \backslash J_{Y}$.

If $J_{Y} \cap \bar{J} \neq \emptyset$, then $\mathcal{L}(D(\bar{J}))=0$ since $S_{Y_{0}}^{3}=S^{3}$ when $Y_{0}$ is a $Y$-graph in $S^{3}$ with a trivial leaf.

If $\overline{J_{Y}} \cap J \neq \emptyset$, then $\ell(D(J) ; \Gamma)=0$ like in the previous case. Then

$$
\ell(D ; \Gamma)= \begin{cases}0 & \text { if } k \neq k^{\prime} \\ \ell\left(D\left(J_{Y}\right) ; \Gamma\right) \cdot \mathcal{L}\left(D\left(\overline{J_{Y}}\right)\right) & \text { if } k=k^{\prime}\end{cases}
$$

Then $\mathcal{L}\left(D\left(\overline{J_{Y}}\right)\right)=1$ by Lemma 4.3 and $\ell\left(D\left(J_{Y}\right) ; \Gamma\right)=\ell\left(\phi_{2 k}\left(\Gamma_{Y}\right) ; \Gamma\right)$. Thus the result follows from the first case. 


\subsection{Decomposition of LP-surgeries into surgeries on $Y$-links}

In this subsection, we recall known facts and state useful lemmas about the theory of Borromeo surgeries Mat, GGP. We shall see all these facts as consequences of the following single lemma 4.4. As an application of the theory of Borromeo surgeries, we shall prove Proposition 3.1 .

Lemma 4.4 GGP Lemma 2.1] Let $M$ be an oriented 3-manifold (with possible boundary). Let $G$ be a $Y$-graph in $M$ with a trivial leaf that bounds a disc $D$ in $M \backslash G$. Then

- for any framed graph $T_{0}$ in $M \backslash G$ that does not meet $D$, the pair $\left(M_{G}, T_{0}\right)$ is diffeomorphic to the pair $\left(M, T_{0}\right)$.

- If $T$ is a framed graph in $M \backslash G$ that meets $\operatorname{Int}(D)$ at exactly one point, then the pair $\left(M_{G}, T\right)$ is diffeomorphic to the pair $\left(M, T_{G}\right)$, where $T_{G}$ is the framed graph in $M$ presented in Figure 4.

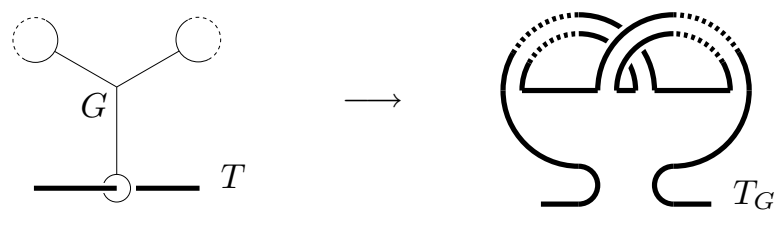

Figure 4

Corollary 4.5 Let $M$ be an oriented 3-manifold. Let $\Sigma$ denote a genus 1 surface in $M$. Let $I_{1}$ and $I_{2}$ be two intervals such that

- $\partial \Sigma=I_{1} \cup I_{2}$

- $I_{1} \cap I_{2}=\partial I_{1}=\partial I_{2}$

- $I_{1}$ and $I_{2}$ are framed by a vector field normal to the surface $\Sigma$.

Let $T$ be a framed graph such that $I_{1}=T \cap \Sigma$. Then there exists a $Y$-graph $G$ in $M \backslash T$ with a trivial leaf that is a meridian curve of $I_{1}$ such that the pair $\left(M_{G}, T\right)$ is diffeomorphic to the pair $\left(M,\left(T \backslash \operatorname{Int}\left(I_{1}\right)\right) \cup I_{2}\right)$

Lemma 4.6 GGP, Theorem 3.2] Let $\Lambda$ be the $Y$-graph in the 3-handlebody $(N=\Sigma(\Lambda) \times[-1,1])$ presented in Figure 1(a) Then there exists a $Y$-graph $\Lambda^{-1}$ in $N \backslash \Lambda$ such that the $Y$-surgery along $\Lambda \cup \Lambda^{-1}$ does not change $N$. In particular, if $M$ is a 3-manifold then, for any $Y$-graph $G$ in $M$, there exists a $Y$-graph $G^{-1}$ in a regular neighbourhood of $G$ such that $M_{G \cup G^{-1}}=M$. 


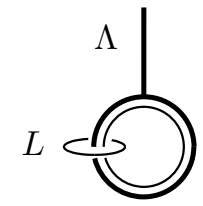

Figure 5: Trivializing a leaf

Proof Let $L$ be the framed link in $N \backslash \Lambda$ made of the two framed knots presented in Figure 5, such that $N_{L}=N$ and such that $\Lambda$ is isotopic to a $Y-$ graph $\Lambda_{0}$ with a trivial leaf in $N_{L}$. Let $L^{-1}$ denote a framed link in $N \backslash(\Lambda \cup L)$ such that the surgery along $L \cup L^{-1}$ is trivial in $N \backslash \Lambda$. Then $L^{-1}$ corresponds to a framed link $L^{\prime}$ in $N_{L}$. Then

$$
N_{\Lambda}=N_{\Lambda \cup L \cup L^{-1}}=\left(N_{L}\right)_{\Lambda_{0} \cup L^{\prime}}
$$

The $Y$-surgery along $\Lambda_{0}$ is fully determined by Lemma 4.4. It takes the tube piercing the trivial leaf and makes it describe its complement in the boundary of a genus one surface.

By Corollary 4.5. there exists a $Y$-graph $\Lambda_{0}^{-1}$ in $N_{L} \backslash\left(\Lambda_{0} \cup L^{\prime}\right)$ that undoes it.

$$
N=\left(\left(N_{L}\right)_{\Lambda_{0} \cup \Lambda_{0}^{-1}}\right)_{L^{\prime}}
$$

After surgery on $L^{\prime}$, that does not change $N$ since the surgery on $L$ did not change $N, \Lambda_{0}^{-1}$ corresponds to a $Y$-graph $\Lambda^{-1}$ in $N \backslash \Lambda$ such that the $Y$-surgery along $\Lambda \cup \Lambda^{-1}$ is trivial.

Remark 4.7 What is used in the above proof and will be used again is the following principle. Up to surgery along links, one leaf of a $Y$-graph can be assumed to bound a disk $D$ (pierced by surgery arcs). Then surgery along that $Y$-graph amounts to move the pack $T$ of framed surgery arcs piercing $D$ as indicated in Lemma 4.4, that therefore fully determines the effect of the surgery along the $Y$-graph.

Lemma 4.8 Let $\phi$ be an embedding of the genus $g$ handlebody $H_{g}$ into $S^{3}$. Let $z_{1}, \ldots, z_{g}$ denote the curves in $\partial H_{g}$ presented in Figure 6. If each curve $\phi\left(z_{i}\right)$ bounds an embedded surface in $S^{3} \backslash \operatorname{Int}\left(\phi\left(H_{g}\right)\right)$, then there exists a $Y$-link $G$ in $S^{3} \backslash \phi\left(H_{g}\right)$ such that $S_{G}^{3}=S^{3}$ and the curves $\phi\left(z_{i}\right)$ bound embedded discs in $S_{G}^{3} \backslash \operatorname{Int}\left(\phi\left(H_{g}\right)\right)$.

Proof Thanks to Corollary 4.5 and to the fact that any orientable surface is a connected sum of genus one surfaces, there exists a $Y$-link $G_{1}$ in $S^{3} \backslash \phi\left(H_{g}\right)$ 


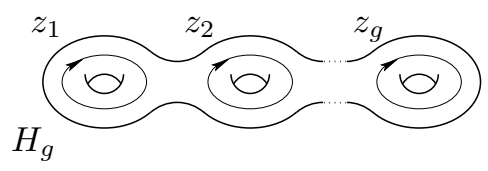

Figure 6

such that $S_{G_{1}}^{3}=S^{3}$ and $\phi\left(z_{1}\right)$ bounds an embedded disc $D_{1}$ in $S^{3} \backslash \phi\left(H_{g}\right)$ after $Y$-surgery on $G_{1}$. In particular the lemma is true for $g=1$. Assume that the lemma is true for handlebodies of genus $g-1$. We shall use this induction hypothesis for a regular neighbourhood $N$ of $\phi\left(H_{g}\right) \cup D_{1}$ equipped with the curves $\phi\left(z_{2}\right), \ldots, \phi\left(z_{g}\right)$ that are still homologically trivial in $S^{3} \backslash N$. Let $\hat{G}_{1} \subset S_{G_{1}}^{3}$ be the union of the 3 -handlebodies reglued during the $Y$-surgery on $G_{1}$. By induction hypothesis, there exists a $Y$-link $G_{2}$ in $S_{G_{1}}^{3} \backslash N$ such that $\left(S_{G_{1}}^{3}\right)_{G_{2}}=S^{3}$ and the curves $\phi\left(z_{2}\right), \ldots, \phi\left(z_{g}\right)$ bound embedded discs in $S^{3} \backslash N$ after $Y$-surgery on $G_{2}$. After a possible isotopy in $S_{G_{1}}^{3}, G_{2}$ avoids $\hat{G}_{1}$. Then $G_{2}$ corresponds to a $Y$-link $G_{2}^{\prime}$ in $S^{3} \backslash\left(\phi\left(H_{g}\right) \cup G_{1}\right)$ such that $G_{1} \cup G_{2}^{\prime}$ satisfies the conclusion of the lemma.

Let $M$ be a $\mathbb{Z}$-sphere. Let $\ell k$ denote the linking number in $M$. Let $L \subset M$ be a link, and let $L_{1}, \ldots, L_{n}$ be the components of $L$. Then $L$ is algebraically split if and only if

$$
(i \neq j) \Rightarrow\left(\ell k\left(L_{i}, L_{j}\right)=0\right) .
$$

Then Lemma 4.8 induces the following corollary.

Corollary 4.9 ([Mat, Lemma 2] or [MN Lemma 1.2]) Let $L$ be an algebraically split link in $S^{3}$. Then there exists a $Y$-link $G$ in $S^{3} \backslash L$ such that $S_{G}^{3}=S^{3}$ and $L$ is trivially embedded in $S_{G}^{3}$.

Proof Embed $H_{g}$ in $S^{3}$ so that the curves $z_{i}$ are the components of $L$.

Theorem 4.10 Mat, Theorem 2] If $M$ and $M^{\prime}$ are homology spheres, then there exists a $Y$-link $G$ in $M$ such that $M_{G}=M^{\prime}$.

Proof Since any $\mathbb{Z}$-sphere can be obtained by surgery on $S^{3}$ along an algebraically split link framed by \pm 1 (see [GM, Lemma 2.1]), and since the surgery on the trivial knot in $S^{3}$ framed by \pm 1 gives $S^{3}$, Theorem 4.10 is an easy corollary of Lemma 4.6 and Corollary 4.9

Then we can prove the following useful lemma (see Hbg, Theorem 2.5], too). 
Lemma 4.11 Let $A$ and $B$ be two $\mathbb{Z}$-handlebodies with the same genus, whose boundaries are identified so that $\mathcal{L}_{A}=\mathcal{L}_{B}$. Then there exists a $Y$-link $G$ embedded in the interior of $A$ such that $A_{G}=B$, where the identification of $\partial A$ with $\partial B$ is induced by the natural identification of $\partial A$ with $\partial A_{G}$.

Proof Let us first prove the lemma when $B=H_{g}$ is the standard handlebody of genus $g$ with the boundary of $A$ identified with the boundary $\Sigma_{g}$ of $H_{g}$ so that $\mathcal{L}_{A}=\mathcal{L}_{H_{g}}$. Embed $H_{g}$ trivially in $S^{3}$ so that

$$
\tilde{H}_{g}=S^{3} \backslash \operatorname{Int}\left(H_{g}\right)
$$

is a standard $g$-handlebody. Let $z_{1}, \ldots, z_{g}$ be the meridian curves of $H_{g}$ on $\Sigma_{g}$ presented in Figure 7

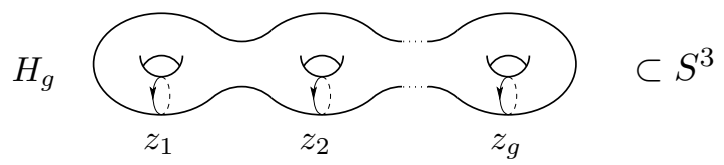

Figure 7

Let

$$
M=\left(S^{3} \backslash \operatorname{Int}\left(H_{g}\right)\right) \cup_{\Sigma_{g}} A=\tilde{H}_{g} \cup_{\Sigma_{g}} A .
$$

Then $M$ is a $\mathbb{Z}$-sphere. Thus, by Theorem 4.10, there exists a $Y$-link $G \subset M$ such that $M_{G}=S^{3}$. By isotopy, $G$ can avoid $\tilde{H}_{g}$. Then

$$
S^{3}=A_{G} \cup_{\Sigma_{g}} \tilde{H}_{g}
$$

Now $A_{G}$ is the complement in $S^{3}$ of a possibly knotted $g$-handlebody $\tilde{H}_{g}$. Thanks to Lemma 4.8, there exists a $Y$-link $G^{\prime} \subset \operatorname{Int}\left(A_{G}\right)$ such that $S_{G^{\prime}}^{3}=S^{3}$ and $\tilde{H}_{g}$ is embedded in $S_{G^{\prime}}^{3}$ so that the curves $z_{i}$ bound embedded discs in $A_{G \cup G^{\prime}}$. Thus $A_{G \cup G^{\prime}}=H_{g}$ with the expected boundary identification. The general case follows easily with the help of Lemma 4.6

We have the following obvious lemma.

Lemma 4.12 Let $D=\left(M ; n ;\left(A_{i}, B_{i}\right)\right)$ be an $n$-component LP-surgery. Let $A_{1}^{\prime}$ be a $\mathbb{Z}$-handlebody such that $\partial A_{1}^{\prime}$ and $\partial A_{1}$ are identified so that $\mathcal{L}_{A_{1}^{\prime}}=$ $\mathcal{L}_{A_{1}}$. Let

$$
M_{A_{1}^{\prime} / A_{1}}=\left(M \backslash \operatorname{Int}\left(A_{1}\right)\right) \cup_{\partial A_{1}} A_{1}^{\prime}
$$

denote the manifold obtained by surgery on $M$ along the pair $\left(A_{1}, A_{1}^{\prime}\right)$. Set

$$
\begin{gathered}
D^{\prime}=\left(M ; n ;\left(A_{1}, A_{1}^{\prime}\right),\left(A_{2}, B_{2}\right), \ldots,\left(A_{n}, B_{n}\right)\right) \\
D^{\prime \prime}=\left(M_{A_{1}^{\prime} / A_{1}} ; n ;\left(A_{1}^{\prime}, B_{1}\right),\left(A_{2}, B_{2}\right), \ldots,\left(A_{n}, B_{n}\right)\right) .
\end{gathered}
$$


Then

$$
[D]=\left[D^{\prime}\right]+\left[D^{\prime \prime}\right]
$$

Proof of Proposition 3.1 Let $D=\left(M ; n ;\left(A_{i}, B_{i}\right)\right)$ be an $n$-component LP-surgery. Thanks to Lemma 4.11, for any $i \in\{1, \ldots, n\}$, there exists a $Y$-link $G^{i} \subset \operatorname{Int}\left(A_{i}\right)$ such that $\left(A_{i}\right)_{G^{i}}=B_{i}$. Let $k_{i}$ denote the minimal number of components for such a $G_{i}$. Consider the sum $k=\sum_{i} k_{i}$.

If there exists $i \in\{1, \ldots, n\}$ such that $k_{i}=0$, then $[D]=0 \in \mathcal{F}_{n}$. If, for all $i$, $k_{i}=1$, then $[D] \in \mathcal{F}_{n}$ by definition. Therefore $[D] \in \mathcal{F}_{n}$ if $k \leq n$.

If $k>n$, assume that $k_{1}>1$, without loss of generality. Then there exists a $\mathbb{Z}$-handlebody $A_{1}^{\prime}$ verifying the hypotheses of Lemma 4.12 such that $A_{1}^{\prime}$ can be obtained from $A_{1}$ by $Y$-surgery along a $Y$-graph in $\operatorname{Int}\left(A_{1}\right)$, and $B_{1}$ can be obtained from $A_{1}^{\prime}$ by $Y$-surgery along a $Y$-link in $\operatorname{Int}\left(A_{1}^{\prime}\right)$ with $k_{1}-1$ components. Thus, with the notation of Lemma 4.12, $\left[D^{\prime}\right] \in \mathcal{F}_{n}$ and $\left[D^{\prime \prime}\right] \in \mathcal{F}_{n}$ by induction on $k$. Then $[D] \in \mathcal{F}_{n}$ thanks to Lemma 4.12, The proposition follows.

\subsection{Review of the clover calculus}

In this section, we review the clover calculus following GGP. However we produce alternative proofs in the spirit of the present paper only based on Lemma 4.4. Furthermore, we summarize what we shall use about the clover calculus in Proposition 4.13 ,

A $Y$-graph $\Lambda$ is oriented if its framing surface $\Sigma(\Lambda)$ is equipped with an orientation. Such an orientation provides an orientation for every leaf and (a cyclic order) for the set of leaves of $\Lambda$. Figure 8 shows the induced orientations when $\Sigma(\Lambda)$ is given the standard orientation of $\mathbb{R}^{2}$. Reversing the orientation of $\Sigma(\Lambda)$ reverses these four orientations.

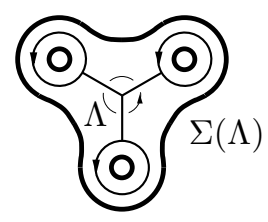

Figure 8: oriented $Y$-graph

Recall that a framing of a knot is a nonzero vector field normal to the knot, up to homotopy, or a parallel to the knot up to isotopy. In a homology sphere 
these two canonically equivalent notions are represented by the linking number of the knot and its parallel induced by the framing. This linking number is therefore also called the framing of the knot.

The goal of this subsection is to prove the following proposition.

Proposition 4.13 Let $G$ be an oriented $n$-component $Y$-link in a $\mathbb{Z}$-sphere $M$.

(i) The bracket $\overline{[(M ; G)]}$ (in $\mathcal{G}_{n}$ ) is a function independent of $M$ of

- the linking numbers $\ell k\left(l, l^{\prime}\right)$ where $l$ and $l^{\prime}$ are leaves in two distinct $Y$-components of $G$

- the products $\bar{f}\left(l_{1}\right) \bar{f}\left(l_{2}\right) \bar{f}\left(l_{3}\right)$ where $l_{1}, l_{2}$ and $l_{3}$ are leaves of a same $Y$-component, and where $\bar{f}(l)$ is the framing of $l$ in $\mathbb{Z} / 2 \mathbb{Z}$.

(ii) Fix $G$ except for a leaf $l$ in the complement of the other parts of $G$. Let [l] denote the class of $l$ in $H_{1}\left(M \backslash \cup_{l^{\prime} \neq l} l^{\prime}\right)$, where the union runs over all leaves $l^{\prime}$ distinct of $l$. Then the bracket $\left(\overline{[(M ; G)]} \in \mathcal{G}_{n}\right)$ of $G$ is a linear map of $([l], \bar{f}(l)) \in H_{1}\left(M \backslash \cup_{l^{\prime} \neq l} l^{\prime}\right) \times \mathbb{Z} / 2 \mathbb{Z}$.

Lemma 4.14 Let $G$ be an oriented $n$-component $Y$-link in a $\mathbb{Z}$-sphere $M$. The bracket $\overline{[(M ; G)]}$ is a function independent of $M$ of

- the linking numbers $\ell k\left(l, l^{\prime}\right)$ where $l$ and $l^{\prime}$ are leaves of $G$

- the framings $f(l)$ where $l$ runs over the leaves of $G$.

Proof Let $\Upsilon$ be the diagram made of $n$ copies of the diagram $\Lambda$ connected by an additional edge from the internal vertex of $\Lambda$ to a common $n$-valent vertex $p$. Embed $\Upsilon$ in $\mathbb{R}^{3}$. Let $A$ be a regular neighbourhood of $\Upsilon$ in $\mathbb{R}^{3}$. Then $A$ is a union of a ball $B$ with $n$ copies of the genus 3 handlebody $N$ that are glued on $\partial B$ along $n$ disjoint discs. Let $\phi_{G}: A \longrightarrow M$ be an embedding of $A$ in $M$ that extends the embedding $G$. Set $Z=M \backslash \operatorname{Int}\left(\phi_{G}(A)\right)$. Then $Z$ is a genus $3 n$ homology handlebody whose Lagrangian $\mathcal{L}_{Z} \subset H_{1}(\partial A)$ is fully determined by the framings and by the linking numbers of the leaves of $G$. Therefore if $G^{\prime} \subset M^{\prime}$ is another oriented $n$-component $Y$-link with the same linking numbers and framing data, then $Z^{\prime}=M^{\prime} \backslash \operatorname{Int}\left(\phi_{G^{\prime}}(A)\right)$ is a homology handlebody with the same lagrangian as $Z$ in $H_{1}(\partial A)$.

By Lemma 4.11 there exists a $Y$-link $G^{\prime \prime} \subset \operatorname{Int}(Z)$ such that $Z_{G^{\prime \prime}}=Z^{\prime}$. Then $\left[\left(M^{\prime} ; G^{\prime}\right)\right]=\left[\left(M_{G^{\prime \prime}} ; G\right)\right]$. If $G^{\prime \prime}$ is a one-component $Y$-link, then $\left[\left(M ; G \cup G^{\prime \prime}\right)\right]=$ $[(M ; G)]-\left[\left(M_{G^{\prime \prime}} ; G\right)\right]$, and $\overline{[(M ; G)]}=\overline{\left[\left(M_{G^{\prime \prime}} ; G\right)\right]}$. By induction on the number of components of $G^{\prime \prime}, \overline{[(M ; G)]}=\overline{\left[\left(M_{G^{\prime \prime}} ; G\right)\right]}$. Then $\overline{[(M ; G)]}=\overline{\left[\left(M^{\prime} ; G^{\prime}\right)\right]}$. 
A framed knot $K_{1} \sharp_{b} K_{2}$ is a band sum of two framed oriented knots $K_{1}$ and $K_{2}$ if there exists an embedding of a 2 -hole disk

- that factors the three knot embeddings by the embeddings of the three curves pictured in Figure 9 representing the disk, and

- that induces the three framings.

Note that

$$
f\left(K_{1} \sharp_{b} K_{2}\right)=f\left(K_{1}\right)+f\left(K_{2}\right)+2 \ell k\left(K_{1}, K_{2}\right) .
$$

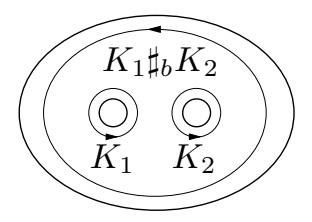

Figure 9: Band sum of two knots

Lemma 4.15 GGP, Theorem 3.1] Let $G$ be an oriented framed $Y$-graph with leaves $K_{1}, K_{2}, K_{3}$ in a $\mathbb{Z}$-sphere $M$. Assume that $K_{3}$ is a band sum of two framed knots $K_{3}^{2}$ and $K_{3}^{3}$. For $k=1$ and 2 , let $K_{k}^{2}$ and $K_{k}^{3}$ be two parallels of $K_{k}$ equipped with the framing $f\left(K_{k}\right)$ of $K_{k}$, and such that $\ell k\left(K_{k}^{2}, K_{k}^{3}\right)=f\left(K_{k}\right)$. Then
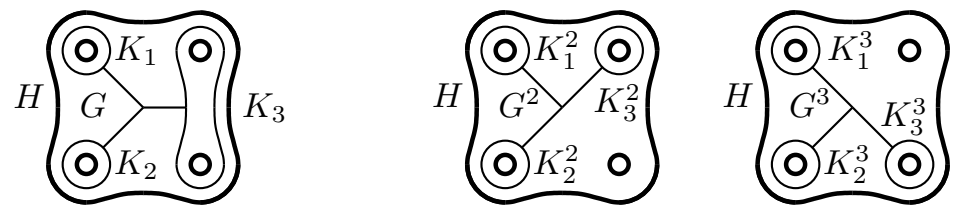

Figure 10 Splitting a leaf

(i) There exist two oriented disjoint framed $Y$-graphs $G^{2}$ and $G^{3}$ in $M$ whose framed leaves are $K_{1}^{2}, K_{2}^{2}, K_{3}^{2}$ and $K_{1}^{3}, K_{2}^{3}, K_{3}^{3}$, respectively, such that the surgery along $G$ is equivalent to the surgery along $G^{2} \cup G^{3}$.

(ii) For any $(n-1)$-component $Y$-link $L$ in the complement in $M$ of the embedded neighbourhood $H$ of $G$ represented in Figure 10,

$$
\overline{[(M ; L \cup G)]}=\overline{\left[\left(M ; L \cup G^{2}\right)\right]}+\overline{\left[\left(M ; L \cup G^{3}\right)\right]} .
$$


Proof The surgery operation on $G$ is thought of as the move of two packs $P_{2}$ and $P_{3}$ of arcs of surgery components that go through the two holes on the right hand-side of $H$ in Figure 10 as in Remark 4.7. Then Lemma 4.4 says that the surgery along $G$ moves these two framed packs of arcs by adding the boundary of a genus 1 surface $\Sigma$. This operation can be made in two steps. Move $P_{3}$ first, which means do the surgery along a $Y$-graph $G^{3}$ whose leaves are $K_{1}^{3}, K_{2}^{3}$ and $K_{3}^{3}$. Then move $P_{2}$ so that it is parallel to $\partial \Sigma$ inside $\Sigma$. It can be done by a surgery along a $Y$-graph $G^{2} \subset H \backslash G^{3}$ whose leaves are $K_{1}^{2}$, $K_{2}^{2}$ and $K_{3}^{2}$. Then $M_{G^{2} \cup G^{3}}=M_{G}$. Therefore

$$
\left[\left(M ; L \cup G^{2} \cup G^{3}\right)\right]=-[(M ; L \cup G)]+\left[\left(M ; L \cup G^{2}\right)\right]+\left[\left(M ; L \cup G^{3}\right)\right]
$$

and

$$
\overline{[(M ; L \cup G)]}=\overline{\left[\left(M ; L \cup G^{2}\right)\right]}+\overline{\left[\left(M ; L \cup G^{3}\right)\right]} .
$$

Lemma 4.16 GGP, Lemma 4.8] Let $G \subset M$ be an $n$-component $Y$-link. Suppose that a $Y$-component of $G$ contains a 2 -framed leaf $l$ that bounds an embedded disc in $M \backslash G$. Then $\overline{[M, G]}=0$.

Proof If $l$ is a 2 -framed leaf that bounds an embedded disc in $M \backslash G$, then $l$ is a band sum of two knots $K^{2}$ and $K^{3}$ that form a trivial Hopf link (see Figure 111) in $M \backslash G$. Thanks to Lemma 4.15, there exist two $n$-components $Y$-links $G^{2}$ and $G^{3}$ with a trivial leaf such that

$$
\overline{[(M ; G)]}=\overline{\left[\left(M ; G^{2}\right)\right]}+\overline{\left[\left(M ; G^{3}\right)\right]}=0 .
$$

Proof of part (ii) of Proposition 4.13 Consider the bracket of $G$ as a function of a leaf $l$ of $G$ by fixing $G \backslash l$. According to Lemma 4.14, the bracket of $G$ only depends on $[l] \in H_{1}\left(M \backslash \cup_{l^{\prime} \neq l} l^{\prime}\right)$ and on $f(l)$. Applying Lemmas 4.15 and 4.16 when adding a disjoint 2 -framed trivial knot to $l$ shows that this function of $l$ only depends on $[l]$ and on $f(l) \bmod 2$. Then Lemma 4.15 implies (ii).

Lemma 4.17 GGP Lemma 2.3] Let $G \subset M$ be an $n$-component $Y$-link. Suppose that $G$ contains a $Y$-component with two leaves $l$ and $l^{\prime}$ that form the trivial Hopf link of Figure [1], Then $\overline{[(M ; G)]}=0$.

Proof The surgery along a $Y$-graph with this trivial Hopf link is trivial: Think of this surgery as the move of surgery arcs along the boundary of the surface corresponding to these two leaves as in Lemma 4.4. It implies that the bracket of $G$ vanishes. 


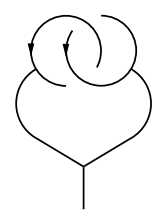

Figure 11: Two leaves that form the trivial Hopf link

Definition 4.18 Let $c$ be a curve in a surface $\Sigma$ that is the image of $S^{1} \times\{\pi\}$ under an orientation-preserving embedding $\phi: S^{1} \times[0,2 \pi] \longrightarrow \Sigma$. A left-handed Dehn twist of $\Sigma$ along $c$ is the homeomorphism of $\Sigma$ that is the identity outside $\phi\left(S^{1} \times\right] 0,2 \pi[)$ and that maps $\phi(z, t)$ to $\phi\left(z e^{-i t}, t\right)$.

Lemma 4.19 GGP, Theorem 3.1] Let $H$ be an oriented $Y$-graph in a $\mathbb{Z}-$ sphere $M$. Let $l^{-}$and $l^{\prime}$ be two oriented leaves of $H$, and let $\Sigma$ be the genus one surface presented in Figure 12, Let $l$ be an oriented parallel of $l^{-}$in $\Sigma$ equipped with the framing induced by $\Sigma$. Let $l^{\prime \prime}$ be obtained from $l^{\prime}$ by a left-handed Dehn twist along $l$, and equipped with the framing induced by the surface $\Sigma$, that is $f\left(l^{\prime \prime}\right)=f(l)+f\left(l^{\prime}\right)+2 \ell k\left(l^{-}, l^{\prime}\right)-1$. Let $H^{\prime}$ be the $Y$-graph obtained from $H$ by changing $l^{\prime}$ into $l^{\prime \prime}$. Then $M_{H}=M_{H^{\prime}}$ and, for any $Y$-link
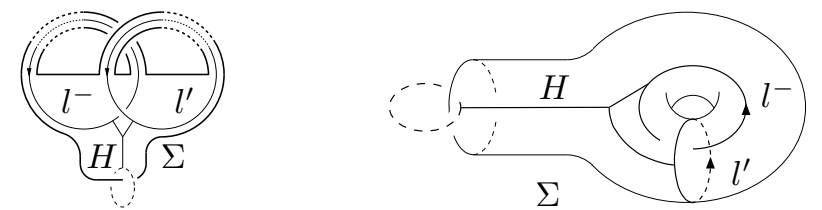

Figure 12

$L$ in the complement in $M$ of a neighbourhood of $H$,

$$
\left[\left(M ; L \cup H^{\prime}\right)\right]=[(M ; L \cup H)] .
$$

Proof Thanks to Lemma 4.4 the surgery on $H$ is uniquely determined by $\Sigma$ that is unchanged by a Dehn twist of $\Sigma$. It implies that $M_{H}=M_{H^{\prime}}$ and that, for any sublink $L(J)$ of $L, M_{L(J) \cup H}=M_{L(J) \cup H^{\prime}}$. The equality of the brackets follows.

\section{End of proof of Proposition 4.13}

- Let $l$ be a leaf of an oriented $Y$-component $H$ of $G$. Let $l^{\prime}$ be the next leaf of $H$ (with respect to the cyclic order). We prove that increasing $\ell k\left(l, l^{\prime}\right)$ does not change $\overline{[(M ; G)]}$. By Lemma 4.15] adding a 0 -framed meridian 
$m_{0}\left(l^{\prime}\right)$ of $l^{\prime}$ to $l$ adds $\overline{\left[\left(M ; G\left(m_{0}\left(l^{\prime}\right) / l\right)\right)\right]}$ to $\overline{[(M ; G)]}$, where $G\left(m_{0}\left(l^{\prime}\right) / l\right)$ is obtained from $G$ by changing $l$ into $m_{0}\left(l^{\prime}\right)$. Now, $l^{\prime}=m^{\prime}+l_{0}^{\prime}$, where $l_{0}^{\prime}$ does not intersect a disk bounded by $m_{0}\left(l^{\prime}\right)$, and $m^{\prime}$ is a meridian of $m_{0}\left(l^{\prime}\right)$. Then

$$
\begin{aligned}
\overline{\left[\left(M ; G\left(m_{0}\left(l^{\prime}\right) / l\right)\right)\right]}= & \overline{\left[\left(M ; G\left(m_{0}\left(l^{\prime}\right), m^{\prime} / l, l^{\prime}\right)\right)\right]} \\
& +\overline{\left[\left(M ; G\left(m_{0}\left(l^{\prime}\right), l_{0}^{\prime} / l, l^{\prime}\right)\right)\right]} \\
= & 0
\end{aligned}
$$

since $G\left(m_{0}\left(l^{\prime}\right), m^{\prime} / l, l^{\prime}\right)$ is a $Y$-link with a trivial Hopf link and since $G\left(m_{0}\left(l^{\prime}\right), l_{0}^{\prime} / l, l^{\prime}\right)$ has a trivial leaf.

- To conclude, it is enough to show that if $l^{-}$is a 0 -framed leaf of $G$, if $l^{\prime}$ is the previous leaf in the component of $l^{-}$in $G$ (w.r.t. the cyclic order), and if $l^{\prime}$ is 1 -framed, then changing the framing of $l^{\prime}$ into 0 does not change $\overline{[(M ; G)]}$. By linearity, we may assume that $l^{\prime}$ is a trivial knot, and then it is enough to show that $\overline{[(M ; G)]}=0$. By linearity on $l^{-}$, we can assume that $l^{-}$is a 0 -framed meridian of some leaf $l_{0}$ in another $Y$-component of $G$. Let $G^{\prime}$ be the $Y$-link obtained from $G$ by changing $l^{\prime}$ into the twisted curve $l^{\prime \prime}=l+l^{\prime}$ as in Lemma 4.19 so that $\bar{f}\left(l^{\prime \prime}\right)=\bar{f}\left(l^{\prime}\right)+1=0$. Then $l^{-}$and $l^{\prime \prime}$ are 0 -framed meridians of $l_{0}$. By linearity with respect to $l_{0}, \overline{\left[\left(M ; G^{\prime}\right)\right]}$ is the sum of brackets of two $Y$-links with a trivial leaf. Then $\overline{\left[\left(M ; G^{\prime}\right)\right]}=\overline{[(M ; G)]}=0$.

\subsection{Proof of Theorem 3.5 for LP-surgeries induced by $Y$-links}

Here we prove Theorem 3.5 when $D=(M ; G)$ is an LP-surgery induced by a $Y$-link $G$.

Lemma 4.20 Let $A$ and $A^{\prime}$ be two $\mathbb{Z}$-handlebodies whose boundaries are identified so that $\mathcal{L}_{A}=\mathcal{L}_{A^{\prime}}$. Let $Z$ and $Z^{\prime}$ be two other $\mathbb{Z}$-handlebodies whose boundaries are identified so that $\mathcal{L}_{Z}=\mathcal{L}_{Z^{\prime}}$. Assume that $\partial A$ and $(-\partial Z)$ are identified so that $A \cup_{\partial A} Z$ is a $\mathbb{Z}$-sphere. Then

$$
\mu\left(A \cup_{\partial A} Z\right)-\mu\left(A^{\prime} \cup_{\partial A} Z\right)=\mu\left(A \cup_{\partial A} Z^{\prime}\right)-\mu\left(A^{\prime} \cup_{\partial A} Z^{\prime}\right) .
$$

Proof For any $\mathbb{Z}$-sphere $M$, let $\lambda(M)$ be the Casson invariant of $M$. Then $\mu(M) \equiv \lambda(M) \bmod 2$. Thanks to [Les, Theorem 1.3],

$$
\lambda\left(A \cup_{\partial A} Z\right)-\lambda\left(A^{\prime} \cup_{\partial A} Z\right)-\left(\lambda\left(A \cup_{\partial A} Z^{\prime}\right)-\lambda\left(A^{\prime} \cup_{\partial A} Z^{\prime}\right)\right)
$$

is an even number. It implies the result. 
Together with Proposition 4.13, it implies

Corollary 4.21 Let $H$ be a $Y$-graph in a $\mathbb{Z}$-sphere $M$. Let

$$
p=\bar{f}\left(l_{1}\right) \bar{f}\left(l_{2}\right) \bar{f}\left(l_{3}\right)
$$

denote the product of the framings of the three leaves of $H$ in $\mathbb{Z} / 2 \mathbb{Z}$. Then

$$
\mu\left(M_{H}\right)-\mu(M)=p .
$$

Proof First, we prove that $\mu$ vanishes on $\mathcal{F}_{2}$. Let $G=G_{1} \cup G_{2}$ be a 2 component $Y$-link in a $\mathbb{Z}$-sphere $M$. Let $A$ be a regular neighbourhood of $G_{1}$. Let $Z$ be the complement of $\operatorname{Int}(A)$ in $M$. Set $A^{\prime}=A_{G_{1}}$ and set $Z^{\prime}=Z_{G_{2}}$. Then $A, A^{\prime}, Z$ and $Z^{\prime}$ satisfy the assumptions of Lemma 4.20 and

$$
\begin{gathered}
\mu\left(M_{G}\right)-\mu\left(M_{G_{1}}\right)-\mu\left(M_{G_{2}}\right)+\mu(M) \\
=\mu\left(A^{\prime} \cup Z^{\prime}\right)-\mu\left(A^{\prime} \cup Z\right)-\mu\left(A \cup Z^{\prime}\right)+\mu(A \cup Z)=0 .
\end{gathered}
$$

Then $\mu\left(M_{H}\right)-\mu(M)$ only depends on $\overline{[(M ; H)]} \in \mathcal{G}_{1}$, thus it only depends on the product $p$ thanks to Proposition 4.13, and it vanishes when $p=0$.

Then $\mu\left(M_{H}\right)-\mu(M)=p$ since $\mu$ is a non-trivial invariant on $\mathcal{F}$.

Lemma 4.22 Let $\tilde{D}=\left(M ; 2 k-1 ;\left(A_{i}, B_{i}\right)_{i=2, \ldots, 2 k}\right)$ be a $(2 k-1)$-component LP-surgery. Let $N$ be a $\mathbb{Z}$-handlebody in $M \backslash\left(\cup_{i=2}^{2 k} A_{i}\right)$. Let $\left(A_{1}, B_{1}\right)$ be a pair of $\mathbb{Z}$-handlebodies such that $A_{1} \subset \operatorname{Int}(N)$ and $\partial A_{1}$ and $\partial B_{1}$ are identified so that $\mathcal{L}_{A_{1}}=\mathcal{L}_{B_{1}}$. Set $D\left(A_{1}, B_{1}\right)=\left(M ; 2 k ;\left(A_{i}, B_{i}\right)\right)$. Let

$$
i_{*}: H_{1}\left(A_{1}\right) \longrightarrow H_{1}(N)
$$

denote the homomorphism induced by the inclusion map of $A_{1}$ into $N$. Set

$$
\mathcal{J}_{1}\left(A_{1}, B_{1}\right)=\left(\otimes^{3}\left(i_{*} \circ \varphi_{A_{1}}^{-1}\right)\right)\left(\mathcal{I}\left(A_{1}, B_{1}\right)\right) \in \bigotimes_{i=1}^{3} H_{1}(N)^{(i)}
$$

where $\varphi_{A_{1}}$ has been defined in Notation 3.3. Then, for any oriented degree $k$ Jacobi diagram $\Gamma$, there exists a linear form $\ell_{N}(\tilde{D} ; \Gamma)$ in $\left(\bigotimes^{3} H_{1}(N)\right)^{*}$ such that, for any pair $\left(A_{1}, B_{1}\right)$ as above,

$$
\ell\left(D\left(A_{1}, B_{1}\right) ; \Gamma\right)=\left\langle\mathcal{J}_{1}\left(A_{1}, B_{1}\right), \ell_{N}(\tilde{D} ; \Gamma)\right\rangle .
$$

Proof Let $\sigma$ be a coloration of $\Gamma$. Set

$$
T^{\prime}(\tilde{D} ; \Gamma ; \sigma)=\operatorname{sign}(h) \bigotimes_{i=2}^{2 k} \mathcal{I}\left(A_{i}, B_{i}\right) \in \otimes_{\{c \in H(\Gamma) ; \sigma \circ v(c)>1\}} X(c) .
$$


Apply all the contractions corresponding to the edges that do not contain any half-edge $c$ in $(\sigma \circ v)^{-1}(1)$. These are all the edges except the three edges $\{c, j(c)\}$ where $c \in(\sigma \circ v)^{-1}(1)$ and $j(c)$ is the other half-edge of $e(c)$, and the obtained tensor is in $\otimes_{\{c \in H(\Gamma) ; \sigma \circ v(c)=1\}} X(j(c))$. Now apply $\bigotimes_{\{c \in H(\Gamma) ; \sigma \circ v(c)=1\}} \varphi_{A_{\sigma \circ v(j(c))}}^{-1}$ in order to obtain the tensor

$$
\ell^{\prime}(\tilde{D} ; \Gamma ; \sigma) \in \bigotimes_{\{c \in H(\Gamma) ; \sigma \circ v(c)=1\}} H_{1}\left(A_{\sigma \circ v(j(c))}\right) .
$$

The linking number maps $H_{1}\left(A_{\sigma \circ v(j(c))}\right)$ to $\left(H_{1}(N)\right)^{*}$ and therefore maps $\ell^{\prime}(\tilde{D} ; \Gamma ; \sigma)$ to an element

$$
\ell_{N}(\tilde{D} ; \Gamma ; \sigma) \in \bigotimes_{i=1}^{3}\left(H_{1}(N)^{*}\right)^{(i)}
$$

By definition, $\ell(\tilde{D} ; \Gamma ; \sigma)$ is the contraction of $\ell_{N}(\tilde{D} ; \Gamma ; \sigma) \otimes \mathcal{J}_{1}\left(A_{1}, B_{1}\right)$. Then

$$
\ell(D ; \Gamma)=\left\langle\mathcal{J}_{1}\left(A_{1}, B_{1}\right), \sum_{\sigma \in \operatorname{Bij}(\Gamma) / \operatorname{Aut}(\Gamma)} \frac{\ell_{N}(\tilde{D} ; \Gamma ; \sigma)}{\sharp \operatorname{Aut}_{V}(\Gamma)}\right\rangle .
$$

Proposition 4.23 Let $n \in \mathbb{N}$. Let $M$ be a $\mathbb{Z}$-sphere. Let $\Gamma$ be an oriented degree $k$ Jacobi diagram with $2 k \leq n$. Consider $\ell((M ; G) ; \Gamma)$ as a function of oriented $n$-component $Y$-links $G$ in $M$. Then

- The linking number $\ell((M ; G) ; \Gamma)$ only depends on

- the linking numbers $\ell k\left(l, l^{\prime}\right)$, where $l$ and $l^{\prime}$ are leaves in two distinct $Y$-components of $G$

- the products $\bar{f}\left(l_{1}\right) \bar{f}\left(l_{2}\right) \bar{f}\left(l_{3}\right)$, where $l_{1}, l_{2}$ and $l_{3}$ are leaves in a same $Y$-component.

- Considered as a map of a leaf $l$ of $G, \ell((M ; G) ; \Gamma)$ is a linear map in $([l], \bar{f}(l)) \in H_{1}\left(M \backslash \cup_{l^{\prime} \neq l^{\prime}} l^{\prime}\right) \times \mathbb{Z} / 2 \mathbb{Z}$.

Proof Assume $n=2 k$. Lemma 4.22 and the expression of $\mathcal{I}\left(A_{1}, B_{1}\right)$ given in Subsection 3.3 show that $\ell((M ; G) ; \Gamma)$ does not depend on the framing of the leaves of $G$ and that $\ell((M ; G) ; \Gamma)$, seen as a map of a leaf $l$ of a component $H$ of $G$, linearly depends on the homology class of $l$ in $M \backslash(G \backslash H)$. It implies the result. If $2 k<n$, the result follows from Corollary 4.21 and from the previous case.

Proposition 4.24 Let $G$ be an $n$-component $Y$-link in a $\mathbb{Z}$-sphere $M$. Then Theorem 3.5 is true when $D=(M ; G)$. 
Proof The simultaneous multilinearities of the bracket in Proposition 4.13 and of the linking number of LP-surgeries induced by $Y$-links in Proposition 4.23 allow us to cut the leaves of $G$ and to reduce the proof in the case where

- The non-zero-framed leaves are \pm 1 -framed and bound discs disjoint from $G$.

- Any 0-framed leaf is a meridian of another leaf.

If a \pm 1 -framed leaf is in a component with a 0 -framed leaf, its framing can be changed without changing either side of the equality. Then we can assume than the only \pm 1 -framed leaves are parts of components like $Y_{I I I}$. Similarly, we can assume that any 0 -framed leaf is a meridian of one leaf in another component of $G$. Then, up to orientation changes of leaves, we can assume that $G$ is a $Y-$ link induced by a Jacobi diagram. Since Theorem 3.5 is true for LP-surgeries induced by Jacobi diagrams, the result follows.

\subsection{Proof of Theorem 3.5] in the general case}

Let $(A, B)$ be a pair of $\mathbb{Z}$-handlebodies whose boundaries $\partial A$ and $\partial B$ are identified so that $\mathcal{L}_{A}=\mathcal{L}_{B}$. In what follows, $\mathcal{I}(A, B)$ denotes the linear form on $\bigotimes^{3} \mathcal{L}_{A}$ induced by the intersection form on $\bigotimes^{3} H_{2}(A \cup(-B))$, and

$$
\varphi_{A}: H_{1}(A) \longrightarrow \mathcal{L}_{A}^{*}
$$

denotes the isomorphism presented in Notation 3.3 .

Lemma 4.25 Let $A, B$ and $C$ be three $\mathbb{Z}$-handlebodies with the same genus. Assume that $\partial A, \partial B$ and $\partial C$ are identified so that $\mathcal{L}_{A}=\mathcal{L}_{B}=\mathcal{L}_{C}$. Then

$$
\mathcal{I}(A, B)=\mathcal{I}(A, C)+\mathcal{I}(C, B) .
$$

Proof Let $a_{1}, a_{2}$ and $a_{3}$ be three oriented curves in $\partial A$ that represent elements of $\mathcal{L}_{A}$ still denoted by $a_{1}, a_{2}$ and $a_{3}$ such that the curves $a_{i}$ do not intersect each other. Let $M=A \cup(-B)$. For any $i \in\{1,2,3\}$, let $S_{A}^{i}$ and $S_{B}^{i}$ be oriented surfaces in $A$ and in $B$, respectively, such that $a_{i}$ bounds $S_{A}^{i}$ in $A$ and $a_{i}$ bounds $S_{B}^{i}$ in $B$. Assume that all the surfaces are transverse to each other and to $\partial A$. Set

$$
\Sigma_{M}^{i}=S_{A}^{i} \cup_{a_{i}}\left(-S_{B}^{i}\right) \subset M .
$$

The orientation of $\Sigma_{M}^{i}$ and the orientation of $M$ induce a positive normal vector field $n_{i}$ on $\Sigma_{M}^{i}$. The algebraic intersection $\left\langle\Sigma_{M}^{1}, \Sigma_{M}^{2}, \Sigma_{M}^{3}\right\rangle_{M}$ is the sum of the signs of the intersection points, where the sign is defined as follows. For any 
intersection point $x$, the sign is +1 if $\left(n_{1}(x), n_{2}(x), n_{3}(x)\right)$ is a direct basis of $T_{x} M$ according to the orientation of $M$, and -1 otherwise. By definition,

$$
(\mathcal{I}(A, B))\left(a_{1} \otimes a_{2} \otimes a_{3}\right)=\left\langle\Sigma_{M}^{1}, \Sigma_{M}^{2}, \Sigma_{M}^{3}\right\rangle_{M} .
$$

Then

$$
\begin{aligned}
(\mathcal{I}(A, B))\left(a_{1} \otimes a_{2} \otimes a_{3}\right) & =\left\langle\Sigma_{M}^{1}, \Sigma_{M}^{2}, \Sigma_{M}^{3}\right\rangle_{M} \\
= & \left\langle S_{A}^{1}, S_{A}^{2}, S_{A}^{3}\right\rangle_{M}+\left\langle\left(-S_{B}^{1}\right),\left(-S_{B}^{2}\right),\left(-S_{B}^{3}\right)\right\rangle_{M} \\
= & \left\langle S_{A}^{1}, S_{A}^{2}, S_{A}^{3}\right\rangle_{A}+\left\langle\left(-S_{B}^{1}\right),\left(-S_{B}^{2}\right),\left(-S_{B}^{3}\right)\right\rangle_{(-B)}
\end{aligned}
$$

Note that the normal vector field $n_{B}^{i}$ to $S_{B}^{i}$ induced by the orientation of $S_{B}^{i}$ and by the orientation of $B$ is equal to the normal vector field to $\left(-S_{B}^{i}\right)$ induced by the orientation of $\left(-S_{B}^{i}\right)$ and by the orientation of $(-B)$. Now, for each point of $S_{B}^{1} \cap S_{B}^{2} \cap S_{B}^{3},\left(n_{B}^{1}, n_{B}^{2}, n_{B}^{3}\right)$ is direct according to the orientation of $B$ if and only if it is not direct according to the orientation of $(-B)$. It implies that

$$
(\mathcal{I}(A, B))\left(a_{1} \otimes a_{2} \otimes a_{3}\right)=\left\langle S_{A}^{1}, S_{A}^{2}, S_{A}^{3}\right\rangle_{A}-\left\langle S_{B}^{1}, S_{B}^{2}, S_{B}^{3}\right\rangle_{B}
$$

and the lemma follows.

Lemma 4.26 Under the hypotheses of Lemma 4.12 for any Jacobi diagram $\Gamma$,

$$
\ell(D ; \Gamma)=\ell\left(D^{\prime} ; \Gamma\right)+\ell\left(D^{\prime \prime} ; \Gamma\right)
$$

Proof The result follows from the equality $\mathcal{I}\left(A_{1}, B_{1}\right)=\mathcal{I}\left(A_{1}, A_{1}^{\prime}\right)+\mathcal{I}\left(A_{1}^{\prime}, B_{1}\right)$ given by Lemma 4.25] and from the equality

$$
\mathcal{L}(D(\{1\}))=\mathcal{L}\left(D^{\prime}(\{1\})\right)+\mathcal{L}\left(D^{\prime \prime}(\{1\})\right) .
$$

Lemma 4.27 Consider an $n$-component $L P$-surgery

$$
D=\left(M ; n ;\left(A_{1}, B_{1}\right),\left(A_{2}, B_{2}\right), \ldots\left(A_{n}, B_{n}\right)\right) .
$$

Let $A_{1}^{\prime}$ and $B_{1}^{\prime}$ be two $\mathbb{Z}$-handlebodies such that

- $A_{1}^{\prime} \subset \operatorname{Int}\left(A_{1}\right)$

- $\partial A_{1}^{\prime}$ and $\partial B_{1}^{\prime}$ are identified so that $\mathcal{L}_{A_{1}^{\prime}}=\mathcal{L}_{B_{1}^{\prime}}$

- $B_{1}=\left(A_{1}\right)_{B_{1}^{\prime} / A_{1}^{\prime}}$ is the $\mathbb{Z}$-handlebody obtained from $A_{1}$ by replacing $A_{1}^{\prime}$ by $B_{1}^{\prime}$.

Set $D^{\prime}=\left(M ; n ;\left(A_{1}^{\prime}, B_{1}^{\prime}\right),\left(A_{2}, B_{2}\right), \ldots,\left(A_{n}, B_{n}\right)\right)$. Then $\left[D^{\prime}\right]=[D]$ while, for any Jacobi diagram $\Gamma$,

$$
\ell\left(D^{\prime} ; \Gamma\right)=\ell(D ; \Gamma)
$$


Sublemma 4.28 Under the hypotheses of Lemma 4.27, let

$$
\begin{array}{lll}
\partial_{A_{1}}: & H_{2}\left(A_{1}, \partial A_{1}\right) & \rightarrow \mathcal{L}_{A_{1}} \\
\partial_{A_{1}^{\prime}}: & H_{2}\left(A_{1}^{\prime}, \partial A_{1}^{\prime}\right) & \rightarrow \mathcal{L}_{A_{1}^{\prime}}
\end{array}
$$

denote the isomorphisms induced by the long exact homology sequences. Let

$$
\begin{array}{ll}
i_{A_{1}}: & H_{2}\left(A_{1}, \partial A_{1}\right) \rightarrow H_{2}\left(A_{1}, A_{1} \backslash \operatorname{Int}\left(A_{1}^{\prime}\right)\right) \\
i_{A_{1}^{\prime}}: & H_{2}\left(A_{1}^{\prime}, \partial A_{1}^{\prime}\right) \rightarrow H_{2}\left(A_{1}, A_{1} \backslash \operatorname{Int}\left(A_{1}^{\prime}\right)\right)
\end{array}
$$

be the homomorphisms induced by the inclusion maps. Then $i_{A_{1}^{\prime}}$ is an isomorphism by the excision axiom. Set

$$
\Phi=\partial_{A_{1}^{\prime}} \circ i_{A_{1}^{\prime}}^{-1} \circ i_{A_{1}} \circ \partial_{A_{1}}^{-1}: \mathcal{L}_{A_{1}} \rightarrow \mathcal{L}_{A_{1}^{\prime}} .
$$

Then

$$
\left(\mathcal{I}\left(A_{1}^{\prime}, B_{1}^{\prime}\right)\right) \circ\left(\otimes^{3} \Phi\right)=\mathcal{I}\left(A_{1}, B_{1}\right)
$$

Proof of Lemma 4.27 assuming Sublemma 4.28 The assertion $\left[D^{\prime}\right]=$ $[D]$ is obvious. Since $\mu\left(M_{B_{1} / A_{1}}\right)=\mu\left(M_{B_{1}^{\prime} / A_{1}^{\prime}}\right)$, it suffices to prove that

$$
\ell(D ; \Gamma)=\ell\left(D^{\prime} ; \Gamma\right)
$$

when $n=2 k$ is even and when $\Gamma$ is a degree $k$ Jacobi diagram. Set $\tilde{D}=$ $\left(M ; 2 k-1 ;\left(A_{i}, B_{i}\right)_{i=2, \ldots, 2 k}\right)$. Let

$$
i_{*}: H_{1}\left(A_{1}^{\prime}\right) \longrightarrow H_{1}\left(A_{1}\right)
$$

be the map induced by the inclusion map of $A_{1}^{\prime}$ into $A_{1}$. By Lemma 4.22 there exists a linear form

$$
\ell_{A_{1}}(\tilde{D} ; \Gamma) \in\left(\stackrel{3}{\bigotimes} H_{1}\left(A_{1}\right)\right)^{*}
$$

such that

$$
\begin{aligned}
& \ell(D ; \Gamma)=\left\langle\left(\otimes^{3} \varphi_{A_{1}}^{-1}\right)\left(\mathcal{I}\left(A_{1}, B_{1}\right)\right), \ell_{A_{1}}(\tilde{D} ; \Gamma)\right\rangle \\
& \ell\left(D^{\prime} ; \Gamma\right)=\left\langle\left(\otimes^{3}\left(i_{*} \circ \varphi_{A_{1}^{\prime}}^{-1}\right)\right)\left(\mathcal{I}\left(A_{1}^{\prime}, B_{1}^{\prime}\right)\right), \ell_{A_{1}}(\tilde{D} ; \Gamma)\right\rangle .
\end{aligned}
$$

The following diagram is commutative

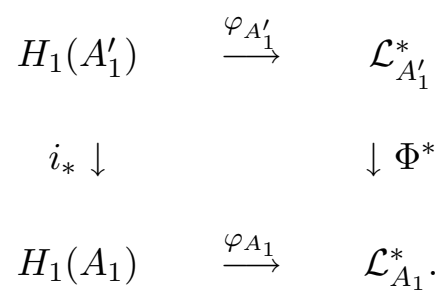


Indeed both compositions, seen as elements of $\left(H_{1}\left(A_{1}^{\prime}\right) \otimes \mathcal{L}_{A_{1}}\right)^{*}$, map $([b],[a]) \in$ $H_{1}\left(A_{1}^{\prime}\right) \times \mathcal{L}_{A_{1}}$ to the algebraic intersection in $A_{1}$ of a surface bounded by $a$ and of the curve $b$. Thus,

$$
\left(\otimes^{3}\left(i_{*} \circ \varphi_{A_{1}^{\prime}}^{-1}\right)\right)\left(\mathcal{I}\left(A_{1}^{\prime}, B_{1}^{\prime}\right)\right)=\left(\otimes^{3} \varphi_{A_{1}}^{-1}\right)\left(\mathcal{I}\left(A_{1}^{\prime}, B_{1}^{\prime}\right) \circ\left(\otimes^{3} \Phi\right)\right) .
$$

By Sublemma 4.28 the lemma follows.

Proof of Sublemma 4.28 Let $a_{1}, a_{2}$ and $a_{3}$ be three oriented curves in $\partial A_{1}$ that represent elements of $\mathcal{L}_{A_{1}}$ still denoted by $a_{1}, a_{2}$ and $a_{3}$ such that the curves $a_{i}$ do not intersect each other. Let $a_{1}^{\prime}, a_{2}^{\prime}, a_{3}^{\prime}$ be oriented curves in $\partial A_{1}^{\prime}$ that represent the elements $\Phi\left(a_{i}\right)$ of $\mathcal{L}_{A_{1}^{\prime}}$ such that the curves $a_{i}^{\prime}$ do not intersect each other. For any $i \in\{1,2,3\}$, the curves $a_{i}$ and $a_{i}^{\prime}$ cobound an oriented surface $\Sigma^{i}$ in $A_{1} \backslash \operatorname{Int}\left(A_{1}^{\prime}\right)$. The curve $a_{i}^{\prime}$ bounds an oriented surface $\sigma_{A_{1}^{\prime}}^{i}$ in $A_{1}^{\prime}$ and bounds an oriented surface $\sigma_{B_{1}^{\prime}}^{i}$ in $B_{1}^{\prime}$. Set

$$
\begin{array}{cccc}
S_{i}= & \left(\sigma_{A_{1}^{\prime}}^{i} \cup \Sigma^{i}\right) \cup\left(-\left(\Sigma^{i} \cup \sigma_{B_{1}^{\prime}}^{i}\right)\right) & \subset & A_{1} \cup\left(-B_{1}\right) \\
S_{i}^{\prime}= & \sigma_{A_{1}^{\prime}}^{i} \cup\left(-\sigma_{B_{1}^{\prime}}^{i}\right) & \subset & A_{1}^{\prime} \cup\left(-B_{1}^{\prime}\right) .
\end{array}
$$

Set

$$
\begin{array}{ccc}
\mathcal{J}_{A_{1}^{\prime} B_{1}^{\prime}} & = & \left(\mathcal{I}\left(A_{1}^{\prime}, B_{1}^{\prime}\right)\right)\left(\Phi\left(a_{1}\right) \otimes \Phi\left(a_{2}\right) \otimes \Phi\left(a_{3}\right)\right) \\
\mathcal{J}_{A_{1} B_{1}} & = & \left(\mathcal{I}\left(A_{1}, B_{1}\right)\right)\left(a_{1} \otimes a_{2} \otimes a_{3}\right) .
\end{array}
$$

By definition, $\mathcal{J}_{A_{1} B_{1}}$ is the intersection in $A_{1} \cup\left(-B_{1}\right)$ of the oriented surfaces $S_{i}$ and $\mathcal{J}_{A_{1}^{\prime} B_{1}^{\prime}}$ is the intersection in $A_{1}^{\prime} \cup\left(-B_{1}^{\prime}\right)$ of the oriented surfaces $S_{i}^{\prime}$. Then $\left(\mathcal{J}_{A_{1} B_{1}}-\mathcal{J}_{A_{1}^{\prime} B_{1}^{\prime}}\right)$ is the contribution of the intersection of the surfaces $\Sigma^{i} \cup\left(-\Sigma^{i}\right)$. This contribution vanishes when $A_{1}^{\prime}=B_{1}^{\prime}$ because $\mathcal{I}\left(A_{1}, A_{1}\right)=$ $\mathcal{I}\left(A_{1}^{\prime}, A_{1}^{\prime}\right)=0$ by Lemma 4.25 Hence it always vanishes.

Proof of Theorem 3.5] Lemmas 4.11, 4.12 and 4.26 allow us to reduce the proof to the case of an LP-surgery $D=\left(M ; n ;\left(A_{i}, B_{i}\right)\right)$ where $B_{i}$ is obtained from $A_{i}$ by a surgery on a $Y$-graph embedded in $A_{i}$. By Lemma 4.27 $D$ can next be considered as an LP-surgery induced by an $n$-component $Y$-link in $M$. Then Theorem 3.5 follows from Proposition 4.24.

\section{A The IHX relation}

For self-containedness, we finish the proof of Theorem 2.1 by proving that $\Psi_{n}$ factors through the IHX relation. This is a consequence of the following proposition. 
Proposition A.1 Let $G_{1}$ be an oriented $Y$-link in a $\mathbb{Z}$-sphere $M$ that admits the following two-component sublink $I_{1}$. For $i=2,3$, let $G_{i}$ be obtained from $G_{1}$ by changing $I_{1}$ into $I_{i}$. The four leaves $a, b, c$ and $d$ are identical.
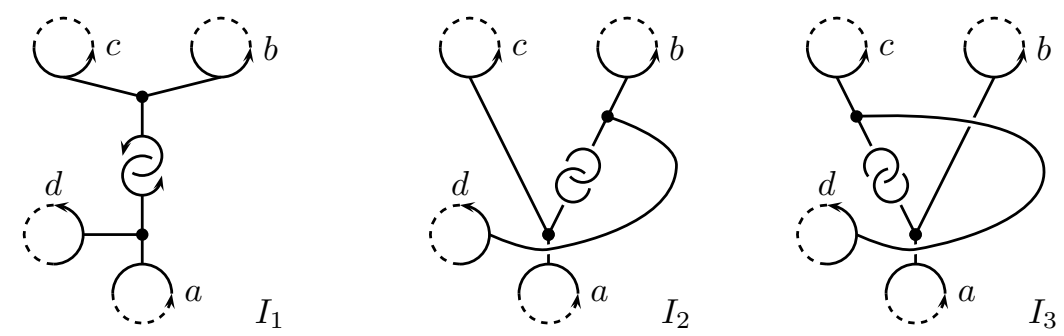

Then

$$
\overline{\left[\left(M ; G_{1}\right)\right]}+\overline{\left[\left(M ; G_{2}\right)\right]}+\overline{\left[\left(M ; G_{3}\right)\right]}=0 .
$$

Proof First recall that Proposition 4.13 implies that the actual embeddings of the $Y$-graph edges do not affect the $Y$-link brackets. Therefore these embeddings will not be specified in the proof below. Consider an embedded product $D_{a b c} \times[0,1]$ of the three-hole disk $D_{a b c}$ whose three inner boundary components are $a, b$ and $c$, and the three generators of $\pi_{1}\left(D_{a b c}\right), \alpha, \beta$ and $\gamma$. We first prove that there exists some two-component $Y$-link $J_{1}$ in $\left(M \backslash\left(G_{1} \backslash I_{1}\right)\right)$ that is obtained from $I_{1}$ by changing (the edge adjacent to $d$ and) the leaves $a, b$ and $c$ into leaves $a(1), b(1)$ and $c(1)$ that are homologous to $a, b$ and $(-c)$ in $\left(M \backslash\left(G_{1} \backslash I_{1}\right)\right)$, respectively, such that surgery on $J_{1}$ makes a pack $T$ of surgery arcs in a surgered disk bounded by $d$ describe the element $\left[\beta \alpha \beta^{-1},[\gamma, \beta]\right]$ of $\pi_{1}\left(D_{a b c}\right)$ in $D_{a b c} \times[0,1]$ in an ascending way with respect to the height of $[0,1]$. Indeed the second part of the following picture shows such a path that is ascending (after sliding two tongues), and that cobounds a genus one surface with the initial shown portion of $T$.
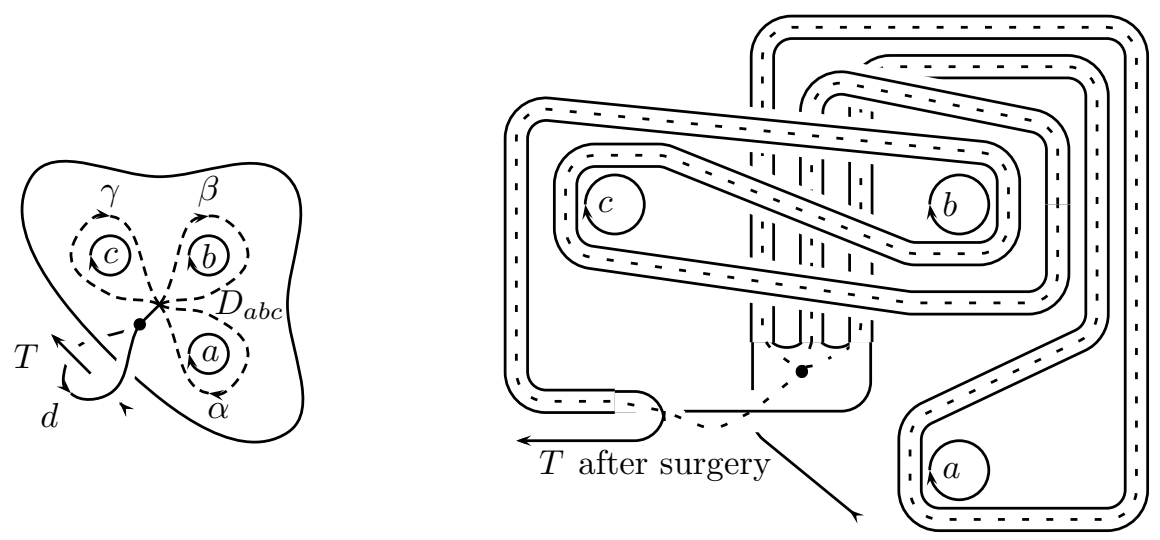
Therefore by Lemma 4.4, this path is obtained from $T$ by surgery on a $Y$-graph with leaves $d, a(1)$ and $f$, where $a(1)$ and $f$ are the two dashed handle cores of the genus one surface, $a(1)$ is homotopic to $\beta \alpha \beta^{-1}$, and $f$ is homotopic to $[\beta, \gamma]$. Thus, $f$ is obtained from a trivial leaf $f_{0}$ by surgery on a $Y$-graph, with one trivial leaf that makes a Hopf link together with $f_{0}$, and two other leaves $b(1)$ and $c(1)$ that do not link $a(1)$ and that are homotopic to $\beta$ and $\gamma^{-1}$, respectively, as Lemma 4.4 and the next picture show.
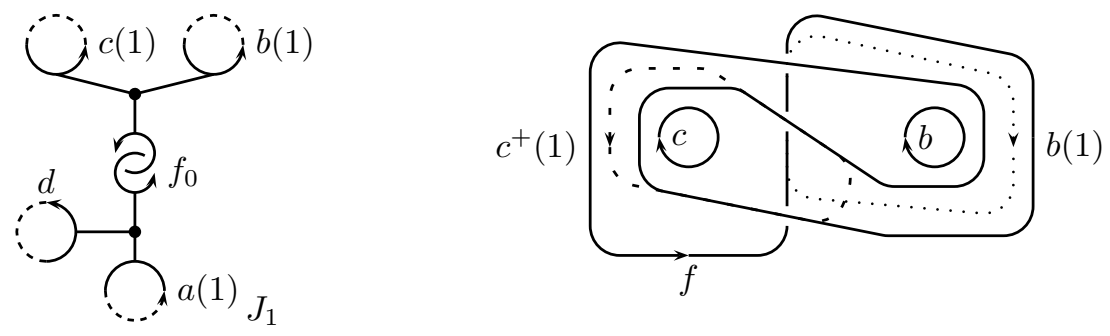

Similarly, for $i=2$ or 3 , there exists a two-component $Y$-link $J_{i}$, that is obtained from $I_{i}$ by changing (the edge adjacent to $d$ and) the leaves $a, b$ and $c$ into leaves $a(i), b(i)$ and $c(i)$ that are homologous to $\alpha(i) a, \beta(i) b$ and $c$ in $\left(M \backslash\left(G_{1} \backslash I_{1}\right)\right)$, respectively, where $\alpha(i), \beta(i) \in\{-1,1\}$, and $\alpha(i) \beta(i)=-1$, such that surgery on $J_{i}$ makes a pack $T$ of surgery arcs in a surgered disk bounded by $d$ describe the element of $\pi_{1}\left(D_{a b c}\right),\left[\gamma \beta \gamma^{-1},[\alpha, \gamma]\right]$ for $i=2$, or $\left[\alpha \gamma \alpha^{-1},[\beta, \alpha]\right]$ for $i=3$, in $D_{a b c} \times[0,1]$ in an ascending way with respect to the height of $[0,1]$.

In particular, the following identity in the free group generated by $\alpha, \beta$ and $\gamma$ -whose verification is straightforward-

$$
\left[\beta \alpha \beta^{-1},[\gamma, \beta]\right]\left[\gamma \beta \gamma^{-1},[\alpha, \gamma]\right]\left[\alpha \gamma \alpha^{-1},[\beta, \alpha]\right]=1
$$

ensures that the surgery on $J_{1} \cup J_{2} \cup J_{3}$ is trivial.

Therefore, if $H_{i}$ is obtained from $G_{i}$ by changing $I_{i}$ into $J_{i}$,

$$
\begin{aligned}
& {\left[M_{J_{1} \cup J_{2}}, H_{3}\right]+\left[M_{J_{1}}, H_{2}\right]+\left[M, H_{1}\right]} \\
& =\sum_{J \subset\left(G_{1} \backslash I_{1}\right)}(-1)^{\sharp J}\left(\begin{array}{l}
M_{J \cup J_{1} \cup J_{2} \cup J_{3}}-M_{J \cup J_{1} \cup J_{2}} \\
+M_{J \cup J_{1} \cup J_{2}}-M_{J \cup J_{1}} \\
+M_{J \cup J_{1}}-M_{J}
\end{array}\right)=0 .
\end{aligned}
$$

Furthermore, Proposition 4.13 ensures that

$$
\overline{\left[\left(M ; G_{i}\right)\right]}=-\overline{\left[\left(M ; H_{i}\right)\right]}=-\overline{\left[\left(M_{\cup_{j<i} J_{j}} ; H_{i}\right)\right]},
$$

and allows us to conclude the proof. 
This proof shows how the Jacobi IHX relation comes from the Lie algebra structure on the graded space associated to the lower central series of a free group. See $\mathrm{MKS}$.

\section{References}

[GGP] S Garoufalidis, M Goussarov, M Polyak, Calculus of clovers and finite type invariants of 3-manifolds, Geom. Topol. 5 (2001) 75-108 MathReview

[GM] L Guillou, A Marin, Notes sur l'invariant de Casson des sphères d'homologie de dimension trois, Enseign. Math. 38 (1992) 233-290 MathReview

[Hbg] N Habegger, Milnor, Johnson, and Tree Level Perturbative Invariants, preprint (2000) http://www.math.sciences.univ-nantes.fr/ habegger

[Hbo] K Habiro, Claspers and finite type invariants of links, Geom. Topol. 4 (2000) 1-83 MathReview

[KT] G Kuperberg, DP Thurston, Perturbative 3-manifold invariants by cutand-paste topology, arXiv:math.GT/9912167

[Le] T T Q Le, An invariant of integral homology 3-spheres which is universal for all finite type invariants, from: "Solitons, geometry, and topology: on the crossroad", Amer. Math. Soc. Transl. 179 (1997) 75-100 MathReview

[Les] C Lescop, A sum formula for the Casson-Walker invariant, Invent. Math. 133 (1998) 613-681 MathReview

[L2] C Lescop, Splitting formulae for the Kontsevich-Kuperberg-Thurston invariant of rational homology 3-spheres, arXiv:math.GT/0411431

[Lic] W B R Lickorish, An Introduction to Knot Theory, Graduate Texts in Mathematics 175, Springer-Verlag, New York (1997) MathReview

[MKS] W Magnus, A Karrass, D Solitar, Combinatorial group theory. Presentations of groups in terms of generators and relations, Second revised edition, Dover Publications, Inc. New York (1976) MathReview

[Mat] S V Matveev, Generalized surgery of three-dimensional manifolds and representations of homology spheres, Mat. Zametki 42 (1987) 268-278, 345 (Russian); English translation: Math. Notes 42 (1987) 651-656 MathReview

[MN] H Murakami, Y Nakanishi, On a certain move generating link-homology, Math. Ann. 284 (1989) 75-89 MathReview

[Oht] T Ohtsuki, Finite type invariants of integral homology 3-spheres, J. Knot Theory Ramifications 5 (1996) 101-115 MathReview

[Rol] D Rolfsen, Knots and Links, Mathematics Lecture Series, No.7. Publish or Perish, Inc. Berkeley, Calif. (1976) MathReview 
[Thu] W P Thurston, The Geometry and Topology of 3-manifolds, Chapter 13, Princeton University (1978) http://www.msri.org/publications/books/gt3m/

Institut Fourier (UMR 5582 du CNRS), B.P. 74 38402 Saint-Martin d'Hères cedex, France

Email: auclaire@ujf-grenoble.fr and lescop@ujf-grenoble.fr

URL: http://www-fourier.ujf-grenoble.fr/ lescop

Received: 9 February 2004 